\title{
CONTINUED FRACTIONS FOR COMPLEX NUMBERS AND VALUES OF BINARY QUADRATIC FORMS
}

\author{
S. G. DANI AND ARNALDO NOGUEIRA
}

\begin{abstract}
We describe various properties of continued fraction expansions of complex numbers in terms of Gaussian integers. Such numerous distinct expansions are possible for a complex number. They can be arrived at through various algorithms, as also in a more general way than what we call "iteration sequences". We consider in this broader context the analogues of the Lagrange theorem characterizing quadratic surds, the growth properties of the denominators of the convergents, and the overall relation between sequences satisfying certain conditions, in terms of non-occurrence of certain finite blocks, and the sequences involved in continued fraction expansions. The results are also applied to describe a class of binary quadratic forms with complex coefficients whose values over the set of pairs of Gaussian integers form a dense set of complex numbers.
\end{abstract}

\section{INTRODUCTION}

Continued fraction expansions of real numbers have been a classical topic in number theory, playing an important role in Diophantine approximation and various other areas. Considerable work has also been done on multi-dimensional versions of the concept. There has however been relatively little work on analogous continued fraction expansion of complex numbers in terms of Gaussian integers. A start was indeed made by Hurwitz in 10 as far back as 1887, where certain properties of the nearest integer algorithm were studied and an analogue of the classical Lagrange theorem for quadratic surds was proved. Some further properties of the Hurwitz continued fraction expansions have been described recently by Hensley 9]. In 11 Leveque explored another direction, focussing primarily on the aspect of relating continued fractions to best approximations in analogy with the classical case. In another work A. Schmidt [13] introduced an entirely different approach to the phenomenon of continued fraction expansions; while the results have some relation with certain classical results, the overall framework is quite different from the classical case. Interesting results have also been obtained by Beardon and Short [2] for continued fraction expansions with partial quotients from convex conical sectors in $\mathbb{C}$ using hyperbolic geometry.

This article is an attempt to explore how far the spirit of classical continued fractions for real numbers can be extended to complex numbers, in terms of the Gaussian integers, viz. $x+i y$ with $x$ and $y$ integers. We view a sequence $\left\{a_{n}\right\}_{n=0}^{\infty}$, of Gaussian integers, with $a_{n} \neq 0$ for $n \geq 1$, as a continued fraction expansion of a complex number $z$ if the corresponding convergents can be formed in the usual way (see below for details) from $a_{0}, a_{1}, \ldots, a_{n}$ for all $n$ in analogy with the usual

Received by the editors March 4, 2011 and, in revised form, August 8, 2012.

2010 Mathematics Subject Classification. Primary 11A55, 11H55; Secondary 22Fxx.

(C) 2014 American Mathematical Society Reverts to public domain 28 years from publication 
convergents, and they converge to $z$. Each complex number has uncountably many distinct continued fraction expansions in this sense.

A class of continued fraction expansions can be generated through algorithms for describing the successive partial quotients, of which the Hurwitz algorithm is an example. Various examples of such algorithms are described in $\S 2$. In $\S 3$ we discuss a more general approach to continued fraction expansions of complex numbers, introducing the notion of "iteration sequences", the terms of which correspond to the tails of continued fraction expansions. We discuss the asymptotics of these sequences in a general framework and prove the convergence of the "convergents" associated with them, which is seen to give a comprehensive picture of the possibilities for continued fractions of complex numbers; see Theorem 3.7

In $\S 4$ we consider continued fraction expansions of quadratic surds, a classical topic for the usual continued fractions. We prove the analogue of the Lagrange theorem in the general framework, and show in particular that for a large class of algorithms (satisfying only a mild condition) the continued fraction expansion of a complex number $z$ is eventually periodic if and only if $z$ is a quadratic surd; see Corollary 4.6 .

Sections 5 and 6 are devoted to the behaviour of the convergents associated to general sequences of partial quotients. In particular we describe conditions on the sequences of partial quotients, in terms of non-occurrence of certain blocks of Gaussian integers, which ensure that the denominators of the convergents have increasing absolute values and exponential growth. This facilitates setting up spaces of sequences together with continuous maps onto the space of irrational complex numbers such that each sequence is a continued fraction expansion of its image under the map; see Theorem 6.1. We also exhibit a space of sequences described purely in terms of non-occurrence of finitely many blocks of length two, each of which is a continued fraction expansion of a complex number, and such that conversely every complex number admits an expansion in terms of such a sequence; see Theorem 6.11

In $\S 7$ we relate the complex continued fractions to fractional linear transformations of the complex plane and the projective space. Associating to each initial block of the continued fraction expansion of a number $z$ a projective transformation in a canonical fashion, we show that the corresponding sequence of transformations asymptotically takes all points of the projective space to the point corresponding to $z$; see Theorem 7.2

Results from $\S 6$ and $\S 7$ are applied in $\S 8$ to study the question of values of binary quadratic forms, with complex coefficients, over the set of pairs of Gaussian integers being dense in complex numbers. It has been known that for a non-degenerate quadratic form in $n \geq 3$ complex variables the set of values over the $n$-tuples of Gaussian integers is dense in complex numbers whenever the form is not a multiple of a form with rational coefficients; see $\S 8$ for some details on the problem. The corresponding statement is however not true for binary quadratic forms. From general dynamical considerations the set of values can be deduced to be dense in $\mathbb{C}$ for "almost all" binary quadratic forms (see $\S 8$ for details), but no specific example is known. We describe a set of complex numbers, called "generic", such that the values of the quadratic form $\left(z_{1}-\varphi z_{2}\right)\left(z_{1}-\psi z_{2}\right)$, where $\varphi, \psi \in \mathbb{C}$, over the set with $z_{1}, z_{2}$ Gaussian integers are dense in $\mathbb{C}$, if $\varphi$ or $\psi$ is generic; see Corollary 8.9. The 
set of generic complex numbers contains a dense $\mathcal{G}_{\delta}$ subset of $\mathbb{C}$, and using a result of D. Hensley [9] it can also be seen to have full Lebesgue measure.

\section{Preliminaries on CONTINUED FRACTION EXPANSIONS}

Let $\mathfrak{G}$ denote the ring of Gaussian integers in $\mathbb{C}$, viz. $\mathfrak{G}=\{x+i y \mid x, y \in \mathbb{Z}\}$. Let $\left\{a_{n}\right\}_{n=0}^{\infty}$ be a sequence in $\mathfrak{G}$. We associate to it two sequences $\left\{p_{n}\right\}_{n=-1}^{\infty}$ and $\left\{q_{n}\right\}_{n=-1}^{\infty}$ defined recursively by the relations

$$
p_{-1}=1, p_{0}=a_{0}, p_{n+1}=a_{n+1} p_{n}+p_{n-1} \text { for all } n \geq 0,
$$

and $\quad q_{-1}=0, q_{0}=1, q_{n+1}=a_{n+1} q_{n}+q_{n-1}$ for all $n \geq 0$.

We call $\left\{p_{n}\right\},\left\{q_{n}\right\}$ the $\mathcal{Q}$-pair of sequences associated to $\left\{a_{n}\right\}$ ( $\mathcal{Q}$ signifies "quotient"). We note that $p_{n} q_{n-1}-q_{n} p_{n-1}=(-1)^{n-1}$ for all $n \geq 0$. If $q_{n} \neq 0$ for all $n$, then we can form the "convergents" $p_{n} / q_{n}$, and if they converge, as $n \rightarrow \infty$ to a complex number $z$, then $\left\{a_{n}\right\}$ can be thought of as a continued fraction expansion of $z$; when this holds we say that $\left\{a_{n}\right\}$ defines a continued fraction expansion of $z$, and write $z=\omega\left(\left\{a_{n}\right\}\right)$. We note that this conforms to the usual idea of continued fraction expansion as $z=a_{0}+\frac{1}{a_{1}+\frac{1}{a_{2}+\cdots}}$ (see [8] for example).

Also, given a finite sequence $\left\{a_{n}\right\}_{n=0}^{m}$, where $m \in \mathbb{N}$, we associate to it sequences $\left\{p_{n}\right\}_{n=-1}^{m}$ and $\left\{q_{n}\right\}_{n=-1}^{m}$ by the recurrence relations as above, and it may also be called the corresponding $\mathcal{Q}$-pair; in this case, provided all $q_{n}$ are non-zero, we set $\omega\left(\left\{a_{n}\right\}\right)=p_{m} / q_{m}$.

Though there will be some occasions to deal with continued fraction expansions of rational complex numbers (namely $z=x+i y$ with $x$ and $y$ rational), by and large we shall be concerned with continued fraction expansions of complex numbers which are not rational (namely $z=x+i y$ with either $x$ or $y$ irrational); this set of complex numbers will be denoted by $\mathbb{C}^{\prime}$.

We note here the following general fact about the convergence of the sequence of the "convergents".

Proposition 2.1. Let $\left\{a_{n}\right\}$ be a sequence in $\mathfrak{G}$ defining a continued fraction expansion of $z \in \mathbb{C}^{\prime}$ and $\left\{p_{n}\right\},\left\{q_{n}\right\}$ be the corresponding $\mathcal{Q}$-pair. Suppose that $\left|q_{n}\right| \geq\left|q_{n-1}\right|$ for all $n \in \mathbb{N}$ and there exist $r \in \mathbb{N}$ and $\theta>1$ such that $\left|q_{n+r}\right| \geq \theta\left|q_{n}\right|$ for all $n \geq 0$. Let $C=r \theta^{2} /\left(\theta^{2}-1\right)$. Then $\left|z-\frac{p_{n}}{q_{n}}\right| \leq C\left|q_{n}\right|^{-2}$ for all $n \in \mathbb{N}$.

Proof. We have $p_{n+1} q_{n}-p_{n} q_{n+1}=(-1)^{n}$ for all $n \geq 0$, so $\frac{p_{n+1}}{q_{n+1}}-\frac{p_{n}}{q_{n}}=\frac{(-1)^{n}}{q_{n} q_{n+1}}$, and in turn $\left|\frac{p_{n+m}}{q_{n+m}}-\frac{p_{n}}{q_{n}}\right| \leq \sum_{k=0}^{m-1} \frac{1}{\left|q_{n+k} q_{n+k+1}\right|}$ for all $n \geq 0$ and $m \geq 1$. Therefore

$$
\begin{gathered}
\left|z-\frac{p_{n}}{q_{n}}\right| \leq \sum_{k=0}^{\infty} \frac{1}{\left|q_{n+k} q_{n+k+1}\right|} \leq \sum_{i=0}^{r-1} \sum_{k=0}^{\infty} \frac{1}{\left|q_{n+k r+i}\right|^{2}} \\
\leq r \sum_{k=0}^{\infty} \frac{\theta^{-2 k}}{\left|q_{n+i}\right|^{2}} \leq \frac{r}{\left|q_{n}\right|^{2}} \sum_{k=0}^{\infty} \theta^{-2 k} \leq \frac{r \theta^{2}}{\left(\theta^{2}-1\right)\left|q_{n}\right|^{2}}=C\left|q_{n}\right|^{-2},
\end{gathered}
$$

which proves the proposition. 
There is considerable flexibility in writing continued fraction expansions of complex numbers, as will become apparent as we proceed. One of the simplest ways of arriving at such expansions is via algorithms for generating a continued fraction expansion starting with a given complex number. Throughout the rest of this section we shall discuss various possibilities in this respect, together with their significance.

We begin by introducing some notation. For any $z \in \mathbb{C}$ and $r>0$ we shall denote by $B(z, r)$ the open disc with center at $z$ and radius $r$, namely $\{\zeta \in \mathbb{C}|| \zeta-z \mid<r\}$, and by $\bar{B}(z, r)$ its closure $\{\zeta \in \mathbb{C}|| \zeta-z \mid \leq r\}$. Furthermore, we shall also write, for $z \in \mathbb{C}, B(z)$ for $B(z, 1), \bar{B}(z)$ for $\bar{B}(z, 1), B$ for $B(0,1)$ and $\bar{B}$ for $\bar{B}(0,1)$. The set of all non-zero complex numbers will be denoted by $\mathbb{C}^{*}$.

Let $f: \mathbb{C}^{*} \rightarrow \mathfrak{G}$ be a map such that $f(z) \in \bar{B}(z)$ for all $z \in \mathbb{C}^{*}$. There are multiple choices possible for the values of $f$ at any point. Using the map we associate to each $z \in \mathbb{C}$ two sequences $\left\{z_{n}\right\}$ and $\left\{a_{n}\right\}$ (finite or infinite) as follows. We define $z_{0}=z, a_{0}=f(z)$ and having defined $z_{0}, \ldots, z_{n}$ and $a_{0}, \ldots, a_{n}, n \geq 0$, we terminate the sequences if $f\left(z_{n}\right)=z_{n}$ and otherwise define $z_{n+1}=\left(z_{n}-f\left(z_{n}\right)\right)^{-1}$ and $a_{n+1}=f\left(z_{n+1}\right)$. We call the map $f$ the choice function of the algorithm, and $\left\{a_{n}\right\}$ the sequence of partial quotients of $z$ with respect to the algorithm (after it is ensured that $\left\{p_{n} / q_{n}\right\}$ converges to $z$ for the corresponding $\mathbb{Q}$-pair $\left\{p_{n}\right\},\left\{q_{n}\right\}$; see Theorem 2.2 below). $\left\{a_{n}\right\}$ may also be called the continued fraction expansion of $z$ with respect to the algorithm, in conformance with the general notion introduced earlier. When the sequences are terminated as above, say with $z_{m}$ and $a_{m}$ where $m \in \mathbb{N}$, then it can be verified inductively that $z=p_{m} / q_{m}$, where $\left\{p_{n}\right\},\left\{q_{n}\right\}$ is the corresponding $\mathcal{Q}$-pair. The crucial case will be when the sequences are infinite, which will necessarily be the case when $z$ is irrational. The following theorem is a particular case of Theorem 3.7

Theorem 2.2. Let $f: \mathbb{C}^{*} \rightarrow \mathfrak{G}$ be the choice function of an algorithm such that $|f(z)-z| \neq 1$ for any $z$ with $|z|=1$. Let $z \in \mathbb{C}^{\prime}$ be given and $\left\{a_{n}\right\}$ be the sequence of partial quotients of $z$ with respect to the algorithm associated with $f$. Let $\left\{p_{n}\right\}$, $\left\{q_{n}\right\}$ be the corresponding $\mathcal{Q}$-pair. Then $q_{n} \neq 0$ for all $n$ and $\left\{p_{n} / q_{n}\right\}$ converges to $z$.

This condition in the theorem is satisfied in particular if we assume at the outset that $f(z) \in B(z)$, in place of $\bar{B}(z)$, for all $z$, but it seems worthwhile to retain the generality, by not making such an assumption.

In some contexts it is convenient to have a slightly more general notion in which the choice function may be allowed to be multivalued over a (typically small, lower dimensional) subset of $\mathbb{C}$, and the algorithm proceeds by picking one of the values. Thus we pick a map $\varphi: \mathbb{C}^{\prime} \rightarrow \mathcal{P}(\mathfrak{G})$ where $\mathcal{P}(\mathfrak{G})$ is the set of subsets of $\mathfrak{G}$, such that $\varphi(z) \subset \bar{B}(z)$ for all $z \in \mathbb{C}^{\prime}$; we choose $z_{0}=z, a_{0} \in \varphi(z)$ and having chosen $z_{0}, \ldots, z_{n}$ and $a_{0}, \ldots, a_{n}, n \geq 0$, we terminate the sequences if $z_{n} \in \varphi\left(z_{n}\right)$ and otherwise define $z_{n+1}=\left(z_{n}-a_{n}\right)^{-1}$ and $a_{n+1} \in \varphi\left(z_{n+1}\right)$. We call $\varphi$ as above a multivalued choice function for an algorithm. One advantage of considering multivalued choice functions $\varphi$ is that these can be chosen to have symmetry properties like $\varphi(\bar{z})=\varphi(z)$ and $\varphi(-z)=\varphi(z)$ for all $z$. Given a multivalued choice function one can get a (single-valued) choice function by auxiliary conventions; in general it may not be possible to ensure the latter to have the symmetry properties.

For the algorithm defined by a choice function $f$ (respectively by a multivalued choice function $\varphi$ ) we call the subset $\overline{\left\{z-f(z) \mid z \in \mathbb{C}^{\prime}\right\}}$ (respectively $\left.\overline{\left\{z-\alpha \mid \alpha \in \varphi(z), z \in \mathbb{C}^{\prime}\right\}}\right)$ the fundamental set of the algorithm, and denote it by 
$\Phi_{f}$ (respectively $\Phi_{\varphi}$ ), or simply $\Phi$. The fundamental sets play a crucial role in the properties of the associated algorithm. We note that if $\Phi$ is contained in $\bar{B}(0, r)$, where $0<r<1$, then $\left|z_{n}\right| \geq r^{-1}>1$ for the iteration sequence corresponding to the algorithm.

Examples 2.3. 1. One of the well-known algorithms is the nearest integer algorithm, discussed in detail by Hurwitz [10, known also as the Hurwitz algorithm. It consists of assigning to $z \in \mathbb{C}$ the Gaussian integer nearest to it; this may be viewed as a multivalued choice function $\varphi: \mathbb{C} \rightarrow \mathcal{P}(\mathfrak{G})$ defined by $\varphi(z)=\{\alpha \in \mathfrak{G}|| z-\alpha \mid \leq$ $|z-\gamma|$ for all $\gamma \in \mathfrak{G}\}$. In this case $\Phi$ is the square $\left\{x+i y|| x\left|\leq \frac{1}{2},\right| y \mid \leq \frac{1}{2}\right\}$.

2. The nearest integer algorithm may be generalized to a class of algorithms as follows: Let $\psi: \mathbb{C}^{*} \rightarrow(0, \infty)$ be a function, and $r \in\left[\frac{1}{\sqrt{2}}, 1\right]$ be given. We define a multivalued choice function $\varphi$ by choosing, for $z \in \mathbb{C}^{\prime}, \varphi(z)$ to be the set of points $x$ in $\bar{B}(z, r) \cap \mathfrak{G}$, where $\psi(x-z)$ is minimum. The nearest integer algorithm is a special case with $\psi(z)=|z|$ for all $z \in \mathbb{C}^{*}$. A class of algorithms $f_{d}$, where $d \in \mathbb{C}$, may be defined by considering the functions defined by $\psi(z)=|z-d|$ in place of $|z|$; we call $\varphi_{d}$ the shifted Hurwitz algorithm with displacement $d$. We note that for the algorithm $\varphi_{1 / 2}$ the continued fraction expansions of real numbers coincide with the usual simple continued fraction expansions. It may also be seen that for $d$ with $|d|<1-\frac{1}{\sqrt{2}}, \Phi_{\varphi_{d}}$ is contained in $\bar{B}\left(0,|d|+\frac{1}{\sqrt{2}}\right)$.

3. Let $f: \mathbb{C}^{*} \rightarrow \mathfrak{G}$ be the map defined as follows: let $z \in \mathbb{C}^{*}$ and $z_{0} \in \mathfrak{G}$ be such that $z-z_{0}=x+i y$ with $0 \leq x<1$ and $0 \leq y<1$; if $x^{2}+y^{2}<1$ we define $f(z)$ to be $z_{0}$ and otherwise $f(z)$ is defined to be $z_{0}+1$ or $z_{0}+i$, whichever is nearer to $z$ (choosing say the former if they are equidistant). It may be seen that with respect to this algorithm, if $z \in \mathbb{C}$ and $\left\{a_{n}\right\}$ is the corresponding sequence of partial quotients, then for all $n \geq 1$ we have $\operatorname{Re} a_{n} \geq-1$ and $\operatorname{Im} a_{n} \geq-1$; this may be compared with the simple continued fractions for real numbers where the latter partial quotients are positive.

4. Let $\mu: \mathfrak{G} \rightarrow(0, \infty)$ be a function on the set of Gaussian integers. Let $\frac{1}{\sqrt{2}} \leq r \leq 1$. A multivalued choice function $\varphi$ for an algorithm may be defined by setting $\varphi(z)$, for $z \in \mathbb{C}^{\prime}$, to be the subset of $\bar{B}(z, r) \cap \mathfrak{G}$ consisting of points where the value of $\mu$ over the set is maximum.

5. One may also restrict the range of the choice function to a subset of $\mathfrak{G}$. If $\mathfrak{G}^{\prime}$ is a subset of $\mathfrak{G}$ such that for some $r \leq 1, B(z, r) \cap \mathfrak{G}^{\prime}$ is non-empty for all $z \in \mathbb{C}^{\prime}$, then choice functions $f$ can be constructed with values in $\mathfrak{G}^{\prime}$, by adopting any of the above strategies. Here is an example. We shall say that $x+i y \in \mathfrak{G}$ is even if $x+y$ is even. Let $\mathfrak{G}^{\prime}$ be the set of all even elements in $\mathfrak{G}$; it is the subgroup generated by $1+i$ and $1-i$, and one can see that $B(z, r) \cap \mathfrak{G}^{\prime}$ is non-empty for all $z \in \mathbb{C}^{\prime}$. In this case by analogy with the nearest integer algorithm we get the "nearest even integer" algorithm; for this algorithm $\Phi$ is the square with vertices at \pm 1 and $\pm i$.

In $\S 6$ we discuss an algorithm with certain interesting properties, involving the choice of nearest integers as well as nearest odd integers depending on the point.

Proposition 2.4. Let $f: \mathbb{C} \rightarrow \mathfrak{G}$ be a choice function such that $\Phi_{f}$ is contained in $B(0, r)$ for some $0<r<1$. Then for any rational complex number the continued fraction expansion with respect to $f$ terminates.

Proof. Let $z=a / b$ be a rational complex number, where $a, b \in \mathfrak{G}$ and $b \neq 0$, and $\left\{z_{n}\right\}$ and $\left\{a_{n}\right\}$ be the sequences, finite or infinite, as above starting with $z$, with 
respect to the algorithm given by $f$. Let $\left\{p_{n}\right\},\left\{q_{n}\right\}$ be the $\mathcal{Q}$-pair corresponding to $\left\{a_{n}\right\}$. From the recurrence relations it may be seen inductively that $q_{n} z-p_{n}=$ $(-1)^{n}\left(z_{0}-f\left(z_{0}\right)\right)\left(z_{1}-f\left(z_{1}\right)\right) \cdots\left(z_{n}-f\left(z_{n}\right)\right)$ for all $n \geq 0$, as long as it is defined (see the proof of Proposition 3.3 below). The condition in the hypothesis then implies that $\left|q_{n} z-p_{n}\right|<r^{n+1}$ for all $n$ as above. For $n$ such that $r^{n+1} \leq|b|^{-1}$ the condition can hold only if $q_{n} z-p_{n}=0$. From the equality as above this implies that $f\left(z_{n}\right)=z_{n}$ for some $n$, so the sequences terminate. This proves the proposition.

The algorithms discussed above are time-independent "Markovian" in the choice strategy, where $z_{n+1}, n \geq 1$, is chosen depending only on $z_{n}$ (and independent of $\left.z_{m}, 0 \leq m \leq n-1\right)$. For certain purposes it is also of interest to consider "time-dependent" algorithms. An interesting example of this is seen in the work of Leveque [11, where an algorithm is introduced such that the corresponding continued fraction expansions have the best approximation property as in the case of the classical continued fractions; this property is not shared by the nearest integer algorithm (nor perhaps by any other time-independent algorithm). We shall not specifically deal with this aspect. Our discussions in the following sections will however also include the continued fraction expansions arising through this type of algorithm.

\section{ITERATION SEQUENCES}

In this section we discuss another, more general approach to the issue of continued fraction expansions, introducing what we call iteration sequences. In this respect for convenience we restrict ourselves to irrational complex numbers. Let $z \in \mathbb{C}^{\prime}$ be given. We call a sequence $\left\{z_{n}\right\}_{n=0}^{\infty}$ an iteration sequence for $z$ if

$$
z_{0}=z, z_{0}-z_{1}^{-1} \in \mathfrak{G} \text {, and for all } n \geq 1,\left|z_{n}\right| \geq 1 \text { and } z_{n}-z_{n+1}^{-1} \in \mathfrak{G} \backslash\{0\} .
$$

An iteration sequence is said to be degenerate if there exists $n_{0}$ such that $\left|z_{n}\right|=1$ for all $n \geq n_{0}$; it is said to be non-degenerate if it is not degenerate.

We note that for any $z \in \mathbb{C}^{\prime}$, depending on the location of $z$ in the unit square with integral vertices that contains $z$, either 2 or 3 or all of the 4 of the vertices are within distance at most 1 from $z ; z_{1}$ can be chosen so that $\left|z_{1}\right| \geq 1$ and $z_{0}-z_{1}^{-1} \in \mathfrak{G}$ is one of these points, and after the choice of $z_{n}, n \geq 1$, is made, between 2 and 4 choices are possible for $z_{n+1}$, the next element of the iteration sequence. For points on the unit circle there are at least two non-zero points of $\mathfrak{G}$ within distance 1 that satisfy the non-degeneracy condition at each $n$. Thus the sequence can be continued in multiple ways at each stage to get a non-degenerate iteration sequence.

Remark 3.1. Given an algorithm with the choice function $f: \mathbb{C}^{*} \rightarrow \mathfrak{G}$ we get an iteration sequence $\left\{z_{n}\right\}$ by setting $z_{0}=z$ and $z_{n+1}=\left(z_{n}-f\left(z_{n}\right)\right)^{-1}$ for all $n \geq 0$. The class of iteration sequences is however more general than sequences arising from the algorithms.

Remark 3.2. Motivated by the classical simple continued fractions, one might consider stipulating that $\left|z_{n}\right|>1$, for $n \geq 1$. However, as we shall see, allowing $\left|z_{n}\right| \geq 1$ introduces no difficulty, provided we ensure that $z_{n}-z_{n+1}^{-1}$ is non-zero, and this enables somewhat greater generality. Also, since in $\mathbb{C}$ there exist irrational numbers $z$ with $|z|=1$, this generality seems appropriate. 
For each iteration sequence $\left\{z_{n}\right\}$ we get a sequence $\left\{a_{n}\right\}$ in $\mathfrak{G}$, called the corresponding sequence of partial quotients, defined by

$$
a_{n}=z_{n}-z_{n+1}^{-1}, \text { for all } n \geq 0 ;
$$

we note that $a_{n} \neq 0$ for all $n \geq 1$. We shall show that if $z_{n}$ is non-degenerate and $\left\{p_{n}\right\}_{n=-1}^{\infty},\left\{q_{n}\right\}_{n=-1}^{\infty}$ is the $\mathcal{Q}$-pair corresponding to $\left\{a_{n}\right\}$, then $q_{n} \neq 0$ for all $n$ and $p_{n} / q_{n}$ converge to $z$ (Theorem [3.7). Thus corresponding to every non-degenerate iteration sequence we get a continued fraction expansion for $z$. We shall also obtain an estimate for the difference $\left|z-\frac{p_{n}}{q_{n}}\right|$ when the iteration sequence is bounded away from the unit circle (see Proposition 3.3.).

Proposition 3.3. Let $z \in \mathbb{C}^{\prime},\left\{z_{n}\right\}$ be an iteration sequence for $z$ and $\left\{a_{n}\right\}$ be the corresponding sequence of partial quotients. Let $\left\{p_{n}\right\},\left\{q_{n}\right\}$ be the $\mathcal{Q}$-pair of sequences associated to $\left\{a_{n}\right\}$. Then we have the following:

(i) $q_{n} z-p_{n}=(-1)^{n}\left(z_{1} \cdots z_{n+1}\right)^{-1}$ for all $n \geq 0$,

(ii) $z=\frac{z_{n+1} p_{n}+p_{n-1}}{z_{n+1} q_{n}+q_{n-1}}$ for all $n \geq 0$,

(iii) for all $n \geq 0,\left|z-\frac{p_{n}}{q_{n}}\right| \leq \frac{1}{\left|q_{n}\right|^{2}\left|z_{n+1}+\frac{q_{n-1}}{q_{n}}\right|}$; in particular, if $\left\{\left|q_{n}\right|\right\}$ is monotonically increasing and there exists a $\gamma>1$ such that $\left|z_{n}\right| \geq \gamma$ for all $n$, then $\left|z-\frac{p_{n}}{q_{n}}\right| \leq \frac{1}{(\gamma-1)\left|q_{n}\right|^{2}}$

Proof. (i) We shall argue by induction. Note that as $p_{0}=a_{0}, q_{0}=1$ and $z-a_{0}=z_{1}^{-1}$, the statement holds for $n=0$. Now let $n \geq 1$ and suppose by induction that the assertion holds for $0,1, \ldots, n-1$. Then we have $q_{n} z-p_{n}=$ $\left(a_{n} q_{n-1}+q_{n-2}\right) z-\left(a_{n} p_{n-1}+p_{n-2}\right)=a_{n}\left(q_{n-1} z-p_{n-1}\right)+\left(q_{n-2} z-p_{n-2}\right)=$ $(-1)^{n-1}\left(z_{1} \cdots z_{n}\right)^{-1} a_{n}+(-1)^{n-2}\left(z_{1} \cdots z_{n-1}\right)^{-1}=(-1)^{n}\left(z_{1} \cdots z_{n}\right)^{-1}\left(-a_{n}+z_{n}\right)=$ $(-1)^{n}\left(z_{1} \cdots z_{n+1}\right)^{-1}$. This proves (i).

(ii) By (i) we have, for all $n \geq 1$,

$$
z_{n+1}=-\frac{q_{n-1} z-p_{n-1}}{q_{n} z-p_{n}} .
$$

Solving the equation for $z$ we get the desired assertion.

(iii) Using (ii) and the fact that $\left|p_{n-1} q_{n}-p_{n} q_{n-1}\right|=1$ we get

$$
\left|z-\frac{p_{n}}{q_{n}}\right|=\left|\frac{z_{n+1} p_{n}+p_{n-1}}{z_{n+1} q_{n}+q_{n-1}}-\frac{p_{n}}{q_{n}}\right|=\frac{1}{\left|q_{n}\right|^{2}\left|z_{n+1}+\frac{q_{n-1}}{q_{n}}\right|},
$$

from which the final assertion readily follows.

Remark 3.4. In the context of (iii) it may be noted here that we shall later see sufficient conditions on $\left\{a_{n}\right\}$ which ensure that $\left\{\left|q_{n}\right|\right\}$ is monotonically increasing. Also the condition $\left|z_{n}\right| \geq \gamma>1$ arises naturally under various circumstances, and holds in particular for iteration sequences arising from any algorithm whose fundamental set is contained in $B(1)$. It may be mentioned that the estimates we get from (iii) in Proposition 3.3 are rather weak and not really significant from the point of view of Diophantine approximation; in fact even the pigeon hole principle yields better inequalities. The main point about the statement however is the generality in terms of allowed sequences $\left\{a_{n}\right\}$ for which the estimates hold. 
Remark 3.5. Let $R$ be the set of all 12 th roots of unity in $\mathbb{C}$. We note that $R$ is precisely the set of points on the unit circle which are also at unit distance from some non-zero Gaussian integer. (For $\rho= \pm 1$ and $\pm i$ there are three such points each, while for the other 12 th roots there is a unique one.) Thus if $\left\{z_{n}\right\}$ is an iteration sequence for $z \in \mathbb{C}^{\prime}$ and for some $m \in \mathbb{N}$ we have $\left|z_{m}\right|=\left|z_{m+1}\right|=1$, then $z_{m} \in R$. It follows in particular that $\left\{z_{n}\right\}$ is a degenerate iteration sequence if and only if $z_{n} \in R$ for all large $n$. It may also be seen, noting that $\left\{z_{n}\right\}$ is contained in $\mathbb{C}^{\prime}$, that if, for some $m \in N, z_{m} \in R$ and $\left|z_{m+1}\right|=1$, then $z_{m+1}=-z_{m}$.

Towards proving that each non-degenerate iteration sequence of any $z \in \mathbb{C}^{\prime}$ yields a continued fraction expansion of $z$ we first note the following:

Proposition 3.6. Let $z \in \mathbb{C}^{\prime}$ and $\left\{z_{n}\right\}$ be a non-degenerate iteration sequence for z. Then $\lim \sup \left|z_{n}\right|>1$. Consequently, $\left|z_{1} \cdots z_{n}\right| \rightarrow \infty$ as $n \rightarrow \infty$.

Proof. Suppose this is not true. As $\left|z_{n}\right| \geq 1$ for all $n \geq 1$ this means that $\left|z_{n}\right| \rightarrow 1$ as $n \rightarrow \infty$. Then $\left|z_{n}-a_{n}\right|=\left|z_{n+1}\right|^{-1} \rightarrow 1$ as $n \rightarrow \infty$. As $a_{n} \in \mathfrak{G} \backslash\{0\}$ for all $n \geq 1$ the above two conditions imply that $d\left(z_{n}, R\right) \rightarrow 0$ as $n \rightarrow \infty$. Furthermore there exist $\rho_{n} \in R$ such that $\left|z_{n}-\rho_{n}\right| \rightarrow 0$. The conditions $\left|z_{n}-a_{n}\right| \rightarrow 1$ and $\left|z_{n}-\rho_{n}\right| \rightarrow 0$ imply in turn that $\left|a_{n}-\rho_{n}\right| \rightarrow 1$ as $n \rightarrow \infty$. Since $\mathfrak{G}$ and $R$ are discrete we get that $\left|a_{n}-\rho_{n}\right|=1$ for all large $n$, say $n \geq n_{0}$. We note also that for any $\rho \in R$ and $a \in \mathfrak{G}$ such that $|\rho-a|=1$, we have $\rho-a \in R$; this can be seen from an inspection of the possibilities. In particular, $\rho_{n}-a_{n} \in R$ for all $n \geq n_{0}$.

Now for all $n$ let $\zeta_{n}=z_{n}-\rho_{n}$ and $\beta_{n}=\rho_{n}-a_{n}$. Then we have $\beta_{n} \in R$ and $z_{n}-a_{n}=\beta_{n}+\zeta_{n}$, and $\zeta_{n} \rightarrow 0$ as $n \rightarrow \infty$. Then

$$
z_{n+1}=\frac{1}{\beta_{n}+\zeta_{n}}=\frac{1}{\beta_{n}}+\frac{-\zeta_{n}}{\beta_{n}\left(\beta_{n}+\zeta_{n}\right)} .
$$

Note that

$$
\left|\frac{\zeta_{n}}{\beta_{n}+\zeta_{n}}\right| \leq \frac{\left|\zeta_{n}\right|}{1-\left|\zeta_{n}\right|} \rightarrow 0
$$

Since $\beta_{n} \in R$, so is $\beta_{n}^{-1}$, and hence the two relations above imply that, for all large $n$,

$$
\rho_{n+1}=\frac{1}{\beta_{n}} \text { and } \zeta_{n+1}=\frac{-\zeta_{n}}{\beta_{n}\left(\beta_{n}+\zeta_{n}\right)} .
$$

Then, for all large $n$,

$$
\left|\zeta_{n+1}\right|=\left|\frac{\zeta_{n}}{\beta_{n}+\zeta_{n}}\right|=\frac{\left|\zeta_{n}\right|}{\left|z_{n}-a_{n}\right|} \geq\left|\zeta_{n}\right|
$$

since $\left|z_{n}-a_{n}\right| \leq 1$. Since $\zeta_{n} \rightarrow 0$ as $n \rightarrow \infty$, this implies that $\zeta_{n}=0$ for all large $n$. Thus $z_{n}=\rho_{n} \in R$ for all large $n$. But this is a contradiction, since $\left\{z_{n}\right\}$ is a non-degenerate iteration sequence; see Remark 3.5. Hence the first assertion in the proposition must hold. The second is a consequence, as $\left|z_{n}\right| \geq 1$ for all $n$.

Theorem 3.7. Let $z \in \mathbb{C}^{\prime},\left\{z_{n}\right\}$ be a non-degenerate iteration sequence for $z$ and $\left\{a_{n}\right\}$ be the corresponding sequence of partial quotients. Let $\left\{p_{n}\right\},\left\{q_{n}\right\}$ be the $\mathcal{Q}$ pair of sequences associated to $\left\{a_{n}\right\}$. Let $n_{0} \geq 0$ be such that $\left|z_{n_{0}+1}\right|>1$. Then $q_{n} \neq 0$ for all $n \geq n_{0},\left|q_{n}\right| \rightarrow \infty$ and $\left\{p_{n} / q_{n}\right\}_{n_{0}}^{\infty}$ converges to $z$ as $n$ tends to infinity. In particular, if $\left|z_{1}\right|$ or $\left|z_{2}\right|$ is greater than 1 , then $q_{n} \neq 0$ for all $n \geq 0$ and $\left\{a_{n}\right\}$ defines a continued fraction expansion for $z$. 
Proof. By Proposition 3.3(i) we have $q_{n} z-p_{n}=(-1)^{n}\left(z_{1} \cdots z_{n+1}\right)^{-1}$ for all $n \geq 0$. Under the condition in the hypothesis we get that $0<\left|q_{n} z-p_{n}\right|<1$ for all $n \geq n_{0}$. Since $p_{n} \in \mathfrak{G}$, this shows in particular that $q_{n} \neq 0$ for all $n \geq n_{0}$. Now suppose there exists a sequence $\left\{n_{k}\right\}$ in $\mathbb{N}$ such that $n_{k} \rightarrow \infty$ as $n \rightarrow \infty$, such that $\left\{q_{n_{k}}\right\}_{k=0}^{\infty}$ is bounded, say $\left|q_{n_{k}}\right| \leq M$ for all $k$. Then $\left|p_{n_{k}}\right| \leq(M+1) /|z|$ and hence it follows that $\left\{p_{n_{k}} / q_{n_{k}} \mid k \in \mathbb{N}\right\}$ is a finite set. Since $\left|q_{n_{k}} z-p_{n_{k}}\right|=\left|z_{1} \cdots z_{n_{k}+1}\right|^{-1} \rightarrow 0$ as $k \rightarrow \infty$, by Proposition 3.6, this implies that $z=p / q$ for some $p, q \in \mathfrak{G}$. This is a contradiction, since $z \in \mathbb{C}^{\prime}$, and hence we get that $\left|q_{n}\right| \rightarrow \infty$ as $n \rightarrow \infty$. Also, $\left|z-\frac{p_{n}}{q_{n}}\right|=\left|z_{1} \cdots z_{n+1}\right|^{-1}\left|q_{n}\right|^{-1} \rightarrow 0$, as $n \rightarrow \infty$. The second part readily follows from the first, noting that $q_{0}=1$. This proves the theorem.

\section{LAGRANGE THEOREM FOR CONTINUED FRACTIONS}

In this section we prove an analogue of the classical Lagrange theorem for the usual simple continued fraction expansions, that the expansion is eventually periodic if and only if the number is a quadratic surd. We shall continue to follow the same notation as before.

A number $z \in \mathbb{C}$ is called a quadratic surd if it is not rational and satisfies a quadratic polynomial over $\mathfrak{G}$, the ring of Gaussian integers; namely, if there exist $a, b, c \in \mathfrak{G}$, with $a \neq 0$, such that $a z^{2}+b z+c=0$. It may be noted that since $z$ is irrational such a polynomial is necessarily irreducible over $\mathfrak{G}$ and both its roots are irrational.

Now, through the rest of the section let $z \in \mathbb{C}^{\prime}$ and $\left\{z_{n}\right\}_{n=0}^{\infty}$ be an iteration sequence for $z$. Also, as before let $a_{n}=z_{n}-z_{n+1}^{-1} \in \mathfrak{G}$, for all $n \geq 0$, and $\left\{p_{n}\right\}$, $\left\{q_{n}\right\}$ be the corresponding $\mathcal{Q}$-pair.

Proposition 4.1. If $z_{m}=z_{n}$ for some $0 \leq m<n$, then $z$ is a quadratic surd.

Proof. By Proposition 3.3(ii) we have

$$
z_{m}=-\frac{z q_{m-2}-p_{m-2}}{z q_{m-1}-p_{m-1}} \text { and } z_{n}=-\frac{z q_{n-2}-p_{n-2}}{z q_{n-1}-p_{n-1}} .
$$

It follows in particular that if $z_{m}$ is a quadratic surd, then so is $z$. We may therefore suppose that $m=0$, so $z_{m}=z$, and $n \geq 1$. The condition $z_{n}=z$ yields, from the above relation, that $z\left(z q_{n-1}-p_{n-1}\right)+z q_{n-2}-p_{n-2}=0$, that is, $q_{n-1} z^{2}+\left(q_{n-2}-p_{n-1}\right) z-p_{n-2}=0$. If $q_{n-1} \neq 0$, then this shows that $z$ is a quadratic surd and we are through. Now suppose that $q_{n-1}=0$. Since $q_{0}=1$, this means that $n \geq 2$. Also, as $q_{n-1}=0$, by Proposition 3.3 we have $\left|p_{n-1}\right|=\left|z_{1} \cdots z_{n}\right|^{-1}$. Since $p_{n-1} \in \mathfrak{G}$ and $\left|z_{k}\right| \geq 1$ for all $k$, this yields that $\left|z_{k}\right|=1$ for all $k=1, \ldots, n$. Since $n \geq 2$, by Remark 3.5 it follows that $z_{1} \in R$. Furthermore, as it is irrational it must be one of the 12 th roots which is a quadratic surd. Hence $z$ is also a quadratic surd.

We now prove the following partial converse of this.

Theorem 4.2. Let $z$ be a quadratic surd. Then for given $C>0$ there exists a finite subset $F$ of $\mathbb{C}$ such that the following holds: if $\left\{z_{n}\right\}$ is a non-degenerate iteration sequence for $z$, with the corresponding $\mathcal{Q}$-pair $\left\{p_{n}\right\},\left\{q_{n}\right\}$, and $N$ is an infinite subset of $\mathbb{N}$ such that for all $n \in N$,

$$
\left|q_{n-2} z-p_{n-2}\right| \leq C\left|q_{n-2}\right|^{-1} \text { and }\left|q_{n-1} z-p_{n-1}\right| \leq C\left|q_{n-1}\right|^{-1},
$$

then there exists $n_{0}$ such that $\left\{z_{n} \mid n \in N, n \geq n_{0}\right\}$ is contained in $F$. 
Proof. Let $a, b, c \in \mathfrak{G}$, with $a \neq 0$, be such that $a z^{2}+b z+c=0$. Let $C>0$ be given. Let $\mathcal{P}$ be the set of all quadratic polynomials of the form $\alpha \zeta^{2}+\beta \zeta+\gamma$, in the variable $\zeta$, with $\alpha, \beta, \gamma \in \mathfrak{G}$ such that $|\alpha| \leq C|2 a z+b|+1,|\gamma| \leq C|2 a z+b|+1$ and $\left|\beta^{2}-4 \alpha \gamma\right|=\left|b^{2}-4 a c\right|$. Then $\mathcal{P}$ is a finite collection of polynomials. Let $F$ be the set of all $\zeta \in \mathbb{C}$ such that $P(\zeta)=0$ for some $P \in \mathcal{P}$; then $F$ is finite. Now let $\left\{z_{n}\right\}$ be any iteration sequence for $z$ and $\left\{p_{n}\right\},\left\{q_{n}\right\}$ be the corresponding $\mathcal{Q}$-pair. Then by Proposition 3.3(ii) for all $n \geq 1$,

$$
\begin{gathered}
a\left(\frac{z_{n} p_{n-1}+p_{n-2}}{z_{n} q_{n-1}+q_{n-2}}\right)^{2}+b\left(\frac{z_{n} p_{n-1}+p_{n-2}}{z_{n} q_{n-1}+q_{n-2}}\right)+c=0 \quad \text { or } \\
a\left(z_{n} p_{n-1}+p_{n-2}\right)^{2}+b\left(z_{n} p_{n-1}+p_{n-2}\right)\left(z_{n} q_{n-1}+q_{n-2}\right)+c\left(z_{n} q_{n-1}+q_{n-2}\right)^{2}=0 .
\end{gathered}
$$

We set

$$
\begin{gathered}
A_{n}=a p_{n-1}^{2}+b p_{n-1} q_{n-1}+c q_{n-1}^{2}, \quad C_{n}=A_{n-1} \quad \text { and } \\
B_{n}=2 a p_{n-1} p_{n-2}+b\left(p_{n-1} q_{n-2}+q_{n-1} p_{n-2}\right)+2 c q_{n-1} q_{n-2} .
\end{gathered}
$$

Then we have

$$
A_{n} z_{n}^{2}+B_{n} z_{n}+C_{n}=0 \text { for all } n \geq 1 .
$$

We note that $A_{n} \neq 0$, since otherwise $p_{n-1} / q_{n-1}$ would be a root of the equation $a x^{2}+b x+c=0$ which is not possible, since the roots of the polynomial are irrational. We rewrite each $A_{n}$ as

$$
\begin{aligned}
A_{n} & =\left(a p_{n-1}^{2}+b p_{n-1} q_{n-1}+c q_{n-1}^{2}\right)-q_{n-1}^{2}\left(a z^{2}+b z+c\right) \\
& =a\left(p_{n-1}^{2}-q_{n-1}^{2} z^{2}\right)+b\left(p_{n-1}-q_{n-1} z\right) q_{n-1} \\
& =\left(p_{n-1}-z q_{n-1}\right)\left(a\left(p_{n-1}+z q_{n-1}\right)+b q_{n-1}\right) \\
& =\left(p_{n-1}-z q_{n-1}\right)\left(a\left(p_{n-1}-z q_{n-1}\right)+(2 a z+b) q_{n-1}\right) .
\end{aligned}
$$

By Proposition [3.6 we have $\left|p_{n-1}-z q_{n-1}\right|=\left|z_{1} \cdots z_{n}\right|^{-1} \rightarrow 0$ as $n \rightarrow \infty$, and hence there exists $n_{0}$ such that for all $n \geq n_{0}$ in $N$ we have

$$
\left|A_{n}\right| \leq|2 a z+b|\left|q_{n-1}\right|\left|p_{n-1}-z q_{n-1}\right|+1 \leq C|2 a z+b|+1,
$$

by the condition in the hypothesis. Since $C_{n}=A_{n-1}$ we also get that $\left|C_{n}\right| \leq$ $C|2 a z+b|+1$ for all large $n$ in $N$. An easy computation shows that for all $n \geq 1$, $B_{n}^{2}-4 A_{n} C_{n}=b^{2}-4 a c$. Thus for all $n \geq n_{0}$ in $N, A_{n} z^{2}+B_{n} z+C_{n}$ is a polynomial belonging to $\mathcal{P}$, and since $z_{n}$ is a root of $A_{n} z^{2}+B_{n} z+C_{n}$ we get that $\left\{z_{n} \mid n \in N, n \geq n_{0}\right\}$ is contained in $F$. This proves the theorem.

Theorem 4.2 and Proposition 2.1 imply the following.

Corollary 4.3. Let $z$ be a quadratic surd. Let $\left\{z_{n}\right\}$ be an iteration sequence for $z$, $\left\{a_{n}\right\}$ be the corresponding sequence, in $\mathfrak{G}$, of partial quotients, and $\left\{p_{n}\right\},\left\{q_{n}\right\}$ be the corresponding $\mathcal{Q}$-pair. Suppose that $\left|q_{n+1}\right|>\left|q_{n}\right|$ for all $n \geq 0$ and there exist $r \in \mathbb{N}$ and $\theta>1$ such that $\left|q_{n+r}\right| \geq \theta\left|q_{n}\right|$ for all $n \geq 0$. Then $\left\{z_{n} \mid n \in \mathbb{N}\right\}$ is finite. Consequently, $\left\{a_{n} \mid n \in \mathbb{N}\right\}$ is also finite.

Corollary 4.3 may be viewed as the analogue of the Lagrange theorem in terms of iteration sequences, under a mild condition on the growth of the denominators of the convergents. Note that when successive terms are not arrived at following an algorithm, the sequence $\left\{a_{n}\right\}$ cannot be expected to be eventually periodic; see Corollary 4.6 below for the corresponding result when the sequence is associated with an algorithm. The condition in Corollary 4.3 on the growth of denominators is satisfied under various conditions (see $\S 6$ ). 
Remark 4.4. We have

$$
\left|q_{n} z-p_{n}\right|=\left|q_{n}\left(\frac{z_{n+1} p_{n}+p_{n-1}}{z_{n+1} q_{n}+q_{n-1}}-\frac{p_{n}}{q_{n}}\right)\right|=\frac{1}{\left|z_{n+1} q_{n}+q_{n-1}\right|}
$$

for all $n \in \mathbb{N}$, and hence the condition in the hypothesis of Theorem 4.2 may be expressed as $\left|z_{n}+\frac{q_{n-2}}{q_{n-1}}\right|>\delta$ and $\left|z_{n+1}+\frac{q_{n-1}}{q_{n}}\right|>\delta$, for some $\delta>0$. We may bear in mind here that $\left|z_{n}\right|>1$ for all $n$ while $\left|q_{n-1} / q_{n}\right|$ may typically be expected to be less than 1, as we shall see in later sections (in particular, see Proposition 5.11).

Corollary 4.5. Suppose that the $z$ as above is a quadratic surd. Let $N=\{n \in \mathbb{N} \mid$ $\left.\left|q_{n-1}\right|>\left|q_{n-2}\right|\right\}$. Suppose that there exists $\gamma>1$ such that $\left|z_{n}\right| \geq \gamma$ for all $n \in N$. Then $\left\{z_{n} \mid n \in N\right\}$ is finite; in particular, there exist $m, n \in N, m \neq n$, such that $z_{m}=z_{n}$.

Proof. For $n \in N$ we have $\left|z_{n}+\frac{q_{n-2}}{q_{n-1}}\right| \geq\left|z_{n}\right|-\left|\frac{q_{n-2}}{q_{n-1}}\right|>\gamma-1$. By the observation in Remark 4.4 we get $\left|q_{n-1} z-p_{n-1}\right| \leq C\left|q_{n-1}\right|^{-1}$, with $C=(\gamma-1)^{-1}$. Thus if $n$ and $n+1$ are both contained in $N$, then $\left|q_{n-1} z-p_{n-1}\right| \leq C\left|q_{n-1}\right|^{-1}$ and $\left|q_{n} z-p_{n}\right| \leq C\left|q_{n}\right|^{-1}$. Now suppose that $n \in N$ and $n+1 \notin N$. Then $\left|q_{n}\right| \leq\left|q_{n-1}\right|$. Therefore $\left|q_{n} z-p_{n}\right|=\left|z_{1} \cdots z_{n+1}\right|^{-1}<\left|q_{n-1} z-p_{n-1}\right| \leq C\left|q_{n-1}\right|^{-1} \leq C\left|q_{n}\right|^{-1}$. Thus the condition as in the hypothesis of Theorem 4.2 is satisfied for all $n$ in $N$ as above. The theorem therefore implies that $\left\{z_{n} \mid n \in N\right\}$ is finite. The second assertion follows from this by the fact that $\left|q_{n}\right| \rightarrow \infty$ as $n \rightarrow \infty$ (see Theorem 3.7), and hence $N$ as above is infinite. This proves the corollary.

We now apply the result to continued fraction expansions arising from timeindependent algorithms, say corresponding to a choice function $f$. Let $z \in \mathbb{C}^{\prime}$ and $\left\{z_{n}\right\}$ be the sequence produced from the algorithm. We note that in this case if $z_{n}=z_{m}$ for some $m, n \in \mathbb{N}$, then $z_{n+l}=z_{m+l}$ for all $l \in \mathbb{N}$. We note also that if $\Phi_{f}$ is contained in $B(0, r)$ for some $r<1$, then $\left|z_{n}\right| \geq r^{-1}$ for all $n \geq 1$. Let $\left\{a_{n}\right\}$ be the corresponding continued fraction expansion. The sequence $\left\{a_{n}\right\}$ is said to be eventually periodic if there exist $m, k \in \mathbb{N}$ such that $a_{j+k}=a_{j}$ for all $j \geq m$.

Corollary 4.6. Let $f$ be a choice function such that the fundamental set $\Phi_{f}$ is contained in $B(0, r)$ for some $r<1$. Then $z \in \mathbb{C}^{\prime}$ is a quadratic surd if and only if its continued fraction expansion $\left\{a_{n}\right\}$ with respect to $f$ is eventually periodic.

Proof. Suppose $\left\{a_{n}\right\}$ is eventually periodic, and let $m, k \in \mathbb{N}$ be such that $a_{j+k}=a_{j}$ for all $j \geq m$. Then $z_{m+k}=z_{m}$ and hence, by Proposition 4.1, $z$ is a quadratic surd. Now suppose that $z$ is a quadratic surd. Since $\Phi_{f}$ is contained in $B(0, r)$ for some $r<1$, by Corollary 4.5 there exist $0 \leq m<n$ such that $z_{n}=z_{m}$. Let $k=n-m$. Then $z_{m+k}=z_{m}$, and as noted above this implies that $z_{j+k}=z_{j}$ for all $j \geq m$. Since $a_{j}=f\left(z_{j}\right)$ for all $j \geq 0$ we get $a_{j+k}=a_{j}$ for all $j \geq m$. This shows that $\left\{a_{n}\right\}$ is eventually periodic.

Remark 4.7. Let $z \in \mathbb{C}^{\prime}$ be a quadratic surd. There are at least two distinct eventually periodic continued fraction expansions for $z$ arising from two different algorithms, since given one such expansion we can find an algorithm whose entries differ from those of the first; furthermore both algorithms may be chosen such that their fundamental sets are contained in the open ball $B(0)$. Secondly, there are infinitely many eventually periodic expansions for $z$, since given any such expansion it can be altered after any given stage to get a new one, which is also eventually periodic; not all of these may correspond to sequences arising from algorithms. 
Clearly, the set of these eventually periodic expansions is countable, since each of them is determined by two finite blocks of Gaussian integers (one corresponding to an initial block and another occurring repeatedly in succession). Recall that for any $z$ there are uncountably many continued fraction expansions (see Remark 3.4). Therefore there are also uncountably many continued fraction expansions which are not eventually periodic.

Remark 4.8. We note that the eventually periodic continued fraction expansions of a quadratic surd corresponding to two different algorithms may differ in their eventual periodicity and also in respect to whether or not the expansion is purely periodic (periodic from the beginning). For example, for $\frac{\sqrt{5}+1}{2}$ we have the usual expansion $\left\{a_{n}\right\}$ with $a_{n}=1$ for all $n$, which may also be seen to be its continued fraction expansion with respect to any of the shifted Hurwitz algorithms $f_{d}$ (see item 2 in Example 2.3 in $\S 2)$, for any real number $d$ in the interval $\left(\frac{\sqrt{5}-1}{2}-\frac{1}{2}, 1\right)$. We recall that from among these, for $d \in\left(\frac{\sqrt{5}-1}{2}-\frac{1}{2}, 1-\frac{1}{\sqrt{2}}\right)$ the associated set $\Phi_{f}$ is contained in $B(0, r)$ for some $r<1$, thus satisfying the condition in Corollary 4.6. On the other hand, the continued fraction expansion of $\frac{\sqrt{5}+1}{2}$ with respect to the Hurwitz algorithm may be seen to be given by $\left\{a_{n}\right\}$, where $a_{0}=2$ and $a_{n}=(-1)^{n} 3$ for all $n \geq 1$; it is not purely periodic, and the eventual period is 2 , rather than 1 .

\section{Growth Properties of the CONVERGents}

In this section we discuss various growth properties of the sequences $\left\{q_{n}\right\}$ from the $\mathcal{Q}$-pairs. We begin with the following general fact.

Proposition 5.1. Let $\left\{a_{n}\right\}_{n=0}^{m}$, where $m \in \mathbb{N}$, be a sequence in $\mathfrak{G}$ such that $\left|a_{n}\right|>1$ for all $n \geq 1$ and let $\left\{p_{n}\right\},\left\{q_{n}\right\}$ be the corresponding $\mathcal{Q}$-pair. Suppose that $q_{n} \neq 0$ for all $n \geq 0$. Let $\mu \in\left(\sqrt{2}, \frac{\sqrt{5}+1}{2}\right)$, and $1 \leq n \leq m-1$ be such that $\left|q_{n-1}\right|>\mu\left|q_{n-2}\right|$. Then the following statements hold: i) if $\left|a_{n}\right|>2$, then $\left|q_{n}\right|>\mu\left|q_{n-1}\right|$; ii) if $\left|a_{n}\right| \leq 2$ and $\operatorname{Re} a_{n} a_{n+1} \geq \chi$, where $\chi=2$ if $\left|a_{n+1}\right|=\sqrt{2}$ and 0 otherwise, then $\left|q_{n+1}\right|>\mu\left|q_{n}\right|$.

Proof. Let $r_{k}=q_{k-1} / q_{k}$, for all $k \geq 0$, and $r=\mu^{-1}$. Then $r \in\left(\frac{\sqrt{5}-1}{2}, 1 / \sqrt{2}\right)$. We have $r_{n}=1 /\left(a_{n}+r_{n-1}\right)$, so $\left|a_{n}\right| \leq\left|r_{n-1}\right|+\left|r_{n}\right|^{-1}<r+\left|r_{n}\right|^{-1}$. When $\left|a_{n}\right|>2$ we get $r+\left|r_{n}\right|^{-1}>\left|a_{n}\right| \geq \sqrt{5}>r+r^{-1}$, since $r>\frac{\sqrt{5}-1}{2}$ and $r+r^{-1}$ is a decreasing function in $(0,1)$. Therefore $\left|r_{n}\right|<r$. This proves the first statement. Now suppose that $\left|a_{n}\right| \leq 2$ and $\operatorname{Re} a_{n} a_{n+1} \geq \chi$ where $\chi$ is as above; in particular, Re $a_{n} a_{n+1} \geq 0$.

Before continuing with the proof we note that for any $r>0$ and $z \in \mathbb{C}$ such that $|z|>r$ the set $B(z, r)^{-1}:=\left\{\zeta^{-1} \mid \zeta \in B(z, r)\right\}$ of the inverses of elements from $B(z, r)$ is precisely $B\left(\frac{\bar{z}}{|z|^{2}-r^{2}}, \frac{r}{|z|^{2}-r^{2}}\right)$.

Now, as $r_{n-1} \in B(0, r)$ and $r<\left|a_{n}\right|$, we get that $r_{n}$, which is $1 /\left(a_{n}+r_{n-1}\right)$, is contained in $B\left(a_{n}, r\right)^{-1}=B\left(y \bar{a}_{n}, y r\right)$, where $y=1 /\left(\left|a_{n}\right|^{2}-r^{2}\right)$. We have $r<1 / \sqrt{2}$, so $1 /(\sqrt{2}-r)<1 /(\sqrt{2}-1 / \sqrt{2})=\sqrt{2}$. Since $\left|a_{n}\right|$ and $\left|a_{n+1}\right|$ are at least $\sqrt{2}$, this shows that $\left|a_{n+1}\right|>1 /\left(\left|a_{n}\right|-r\right)$, which is the same as $y\left(\left|a_{n}\right|+r\right)$. Therefore $\mid y \bar{a}_{n}+$ $a_{n+1}|\geq y| a_{n}|-| a_{n+1} \mid>y r$. We have $r_{n+1}=1 /\left(a_{n+1}+r_{n}\right) \in B\left(a_{n+1}+y \bar{a}_{n}, y r\right)^{-1}$, and in light of the observation above it follows that $r_{n+1} \in B\left(\frac{\bar{z}}{|z|^{2}-s^{2}}, \frac{s}{|z|^{2}-s^{2}}\right)$, where $z=y \bar{a}_{n}+a_{n+1}$ and $s=y r$. We would like to conclude that $r_{n+1} \in B(0, r)$, and for this it suffices to show that $\left|\frac{\bar{z}}{|z|^{2}-s^{2}}\right|+\left|\frac{s}{|z|^{2}-s^{2}}\right|<r$, or equivalently that 
$r(|z|-s)>1$. Substituting for $z$ and $s$ and putting $x=y^{-1}$ we see that the above condition is equivalent to $r\left(\left|\bar{a}_{n}+x a_{n+1}\right|-r\right)>x=\left|a_{n}\right|^{2}-r^{2}$ or, equivalently, $r\left|\bar{a}_{n}+x a_{n+1}\right|>\left|a_{n}\right|^{2}$. Squaring both sides and recalling that $r^{2}=\left|a_{n}\right|^{2}-x$ the condition may be written as

$$
\left(\left|a_{n}\right|^{2}-x\right)\left(\bar{a}_{n}+x a_{n+1}\right)\left(a_{n}+x \bar{a}_{n+1}\right)>\left|a_{n}\right|^{4} .
$$

The condition can be readily simplified to

$$
\left|a_{n+1}\right|^{2} x^{2}+(-\alpha+\beta) x-\left|a_{n}\right|^{2}(\beta-1)<0,
$$

where $\alpha=\left|a_{n}\right|^{2}\left|a_{n+1}\right|^{2}$ and $\beta=2 \operatorname{Re} a_{n} a_{n+1}$. The discriminant $\Delta$ of the quadratic polynomial is seen to be $(\alpha+\beta)^{2}-4 \alpha$. Since $\beta=2 \operatorname{Re} a_{n} a_{n+1} \geq 0$ and $\alpha \geq 4$, we get that $\Delta \geq 0$.

The (real) roots of the quadratic are given by $\lambda_{ \pm}=(\alpha-\beta \pm \sqrt{\Delta})\left|a_{n}\right|^{2} / 2 \alpha$ and the desired condition as above holds if $x \in\left(\lambda_{-}, \lambda_{+}\right)$. As $r^{2}=\left|a_{n}\right|^{2}-x$ we see that the condition holds if $r^{2}$ is in the interval $\left(\left|a_{n}\right|^{2}-\lambda_{+},\left|a_{n}\right|^{2}-\lambda_{-}\right)$or, equivalently, if the following inequalities hold:

$$
(2 \alpha)^{-1}\left|a_{n}\right|^{2}(\alpha+\beta-\sqrt{\Delta})<r^{2}<(2 \alpha)^{-1}\left|a_{n}\right|^{2}(\alpha+\beta+\sqrt{\Delta}) .
$$

We note that the last term is at least $(2 \alpha)^{-1}\left|a_{n}\right|^{2} \alpha=\left|a_{n}\right|^{2} / 2 \geq 1$, and hence it is greater than $r^{2}$. It now suffices to prove that the first inequality holds or, equivalently, that

$$
\mu^{2}<(2 \alpha)\left|a_{n}\right|^{-2}(\alpha+\beta-\sqrt{\Delta})^{-1}=\frac{1}{2}\left|a_{n}\right|^{-2}(\alpha+\beta+\sqrt{\Delta}),
$$

as $(\alpha+\beta)^{2}-\Delta=4 \alpha$. Now first suppose that $\left|a_{n+1}\right| \geq 2$. Then, as $\beta \geq 0$, the right hand side is at least $\frac{1}{2}\left|a_{n}\right|^{-2}\left(4\left|a_{n}\right|^{2}+\sqrt{16\left|a_{n}\right|^{4}-16\left|a_{n}\right|^{2}}\right)=2\left(1+\sqrt{1-\left|a_{n}\right|^{-2}}\right) \geq$ $2+\sqrt{2}$, and hence it indeed exceeds $\mu^{2}$. Now suppose $\left|a_{n+1}\right|=\sqrt{2}$. Then we have $\operatorname{Re} a_{n} a_{n+1} \geq 2$. Under these conditions it can be explicitly verified that the right hand expression above exceeds $\left(\frac{\sqrt{5}+1}{2}\right)^{2}=\frac{3+\sqrt{5}}{2}$. For instance, if $a_{n+1}=1+i$, then the only possibilities for $a_{n}$ in $\mathfrak{G}$ with $\left|a_{n}\right| \leq 2$ and Re $a_{n} a_{n+1} \geq 2$ are $1-i, 2$ or $-2 i$ and the values of the expression may be seen to be $2+\sqrt{3}$ in the first case and $(3+\sqrt{7}) / 2$ in the other two cases. The corresponding assertion for other choices of $a_{n+1}$ follows from this by symmetry considerations (with respect to $z \mapsto-z$ and $z \mapsto \bar{z})$, or may be verified directly. Therefore $r^{2}$ is contained in the desired interval and the argument shows that $r_{n+1} \in B(0, r)$. This proves the proposition.

By applying Proposition 5.1 with $\mu$ smaller than $(\sqrt{5}+1) / 2$ we get the following.

Corollary 5.2. Let $\left\{a_{n}\right\}_{n=0}^{m}$ be a sequence in $\mathfrak{G}$ such that $\left|a_{n}\right|>1$ for all $n \geq 1$ and let $\left\{p_{n}\right\},\left\{q_{n}\right\}$ be the corresponding $\mathcal{Q}$-pair. Suppose that $q_{n} \neq 0$ for all $n \geq$ 0 . Let $1 \leq n \leq m-1$ be such that $\left|q_{n-1}\right| \geq\left(\frac{\sqrt{5}+1}{2}\right)\left|q_{n-2}\right|$, and either $\left|a_{n}\right|>2$ or $\operatorname{Re} a_{n} a_{n+1} \geq \chi$, where $\chi=2$ if $\left|a_{n+1}\right|=\sqrt{2}$ and 0 otherwise. Then either $\left|q_{n}\right| /\left|q_{n-1}\right|$ or $\left|q_{n+1}\right| /\left|q_{n}\right|$ is at least $\frac{\sqrt{5}+1}{2}$.

If $\left\{a_{n}\right\}$ is a sequence in $\mathfrak{G}$ corresponding to continued fraction expansion with respect to the Hurwitz algorithm, then for all $n \geq 1$ such that $\left|a_{n}\right| \leq 2$ we have Re $a_{n} a_{n+1} \geq \chi$, where $\chi$ is as in Proposition 5.1 (see [10] or [9]). We thus get the following corollary. 
Corollary 5.3. Let $\left\{a_{n}\right\}$ be a sequence in $\mathfrak{G}$ given by the continued fraction expansion of a complex number with respect to the Hurwitz algorithm, and let $\left\{p_{n}\right\},\left\{q_{n}\right\}$ be the corresponding $\mathcal{Q}$-pair. Then for any $n \geq 1$ either $\left|q_{n}\right| /\left|q_{n-1}\right|$ or $\left|q_{n+1}\right| /\left|q_{n}\right|$ is at least $(\sqrt{5}+1) / 2$.

Proof. We note that the condition in Corollary 5.2 holds for $n=1$, since $q_{-1}=0$. Applying the corollary successively we get that for any $n \geq 1$ one of the ratios $\left|q_{n}\right| /\left|q_{n-1}\right|$ or $\left|q_{n+1}\right| /\left|q_{n}\right|$ is at least $(\sqrt{5}+1) / 2$.

Remark 5.4. Corollary 5.3 strengthens Lemma 5.1 of [9], where the corresponding assertion is proved (for sequences arising from the Hurwitz algorithm) with the constant $\frac{3}{2}$ in place of $\frac{\sqrt{5}+1}{2}$.

As noted earlier, in various contexts it is useful to know whether $\left|q_{n}\right|$ is increasing with $n$. We shall next address the issue and describe conditions under which this is assured. In this respect we first note the following simple fact about certain finite sequences of complex numbers, which will also be used later.

We denote by $\sigma: \mathbb{C} \rightarrow \mathbb{C}$ the reflexion in the $y$-axis, namely the map defined by $\sigma(z)=-\bar{z}$ for all $z \in \mathbb{C}$.

Remark 5.5. In the sequel it would be convenient to keep in mind that for $z \in \mathbb{C}^{\prime}$ with $|z|=\sqrt{2}$ the set $B(z)^{-1}$, consisting of inverses of numbers in the ball $B(z)$, is precisely $B(\bar{z})$, which is also the same as $B(-\sigma(z))$.

Lemma 5.6. Let $z_{0}, z_{1}, \ldots, z_{m+1}$ be non-zero complex numbers such that $\left|z_{0}\right| \leq 1$ and $\left|z_{n}\right|>1$ for $n=1, \ldots, m+1$. For all $n=0, \ldots, m$ let $b_{n}=z_{n}-z_{n+1}^{-1}$ and suppose that $b_{n} \in \mathfrak{G}$ for all $n$ and that $\left|b_{0}\right|>1$. Then $\left|b_{0}\right|=\sqrt{2}$ and at least one of the following conditions holds:

i) $b_{n}=2 \sigma^{n}\left(b_{0}\right)$ for all $n=1, \ldots, m$ and $z_{n} \in \bar{B}\left(\sigma^{n}\left(b_{0}\right)\right)$ for all $n=1, \ldots, m+1$;

ii) there exists $1 \leq n \leq m$ such that $b_{k}=2 \sigma^{k}\left(b_{0}\right)$ for $k=1, \ldots, n-1, b_{n} \neq$ $2 \sigma^{n}\left(b_{0}\right)$ and $\left|b_{n}-\sigma^{n}\left(b_{0}\right)\right|<2$.

Proof. We have $1<\left|b_{0}\right|=\left|z_{0}-z_{1}^{-1}\right| \leq\left|z_{0}\right|+\left|z_{1}\right|^{-1}<2$, and since $b_{0} \in \mathfrak{G}$ we get that $\left|b_{0}\right|=\sqrt{2}$. Also, since $b_{0}=z_{0}-z_{1}^{-1}$ we have $z_{1}^{-1} \in \bar{B}\left(-b_{0}\right)$, and as $\left|b_{0}\right|=\sqrt{2}$ this implies that $z_{1} \in \bar{B}\left(\sigma\left(b_{0}\right)\right)$ (see Remark 5.5). We now suppose that (ii) does not hold, and deduce, inductively, that $b_{n}=2 \sigma^{n}\left(b_{0}\right)$ and $z_{n+1} \in \bar{B}\left(\sigma^{n+1}\left(b_{0}\right)\right)$ for all $n=1, \ldots, m$. We have $\left|b_{1}-\sigma\left(b_{0}\right)\right| \leq\left|z_{1}-\sigma\left(b_{0}\right)\right|+\left|z_{1}-b_{1}\right|=\left|z_{1}-\sigma\left(b_{0}\right)\right|+\left|z_{2}\right|^{-1}<2$. The assumption that (ii) does not hold therefore implies that $b_{1}=2 \sigma\left(b_{0}\right)$. Now $z_{2}^{-1}-2 \sigma\left(b_{0}\right)=z_{2}^{-1}-b_{1}=z_{1} \in \bar{B}\left(\sigma\left(b_{0}\right)\right)$, so by translation $z_{2}^{-1} \in \bar{B}\left(-\sigma\left(b_{0}\right)\right)$, and therefore $z_{2} \in \bar{B}\left(\sigma^{2}\left(b_{0}\right)\right)$. This proves the inductive statement for $n=1$. Now suppose it holds for some $n \leq m-1$. Then $\left|b_{n+1}-\sigma^{n+1}\left(b_{0}\right)\right| \leq\left|z_{n+1}-\sigma^{n+1}\left(b_{0}\right)\right|+$ $\left|z_{n+1}-b_{n+1}\right|=\left|z_{n+1}-\sigma^{n+1}\left(b_{0}\right)\right|+\left|z_{n+2}\right|^{-1}<2$, the first term being bounded by 1 by the inductive assumption. The assumption that (ii) does not hold now implies that $b_{n+1}=2 \sigma^{n+1}\left(b_{0}\right)$. Also, $z_{n+2}^{-1}-2 \sigma^{n+1}\left(b_{0}\right)=z_{n+2}^{-1}-b_{n+1}=z_{n+1} \in$ $\bar{B}\left(\sigma^{n+1}\left(b_{0}\right)\right)$, so by translation $z_{n+2}^{-1} \in \bar{B}\left(-\sigma^{n+1}\left(b_{0}\right)\right)$ and therefore $\bar{B}\left(\sigma^{n+2}\left(b_{0}\right)\right)$. This completes the inductive argument.

By a block $\beta$ of Gaussian integers we mean a finite $l$-tuple $\beta=\left(b_{1}, \ldots, b_{l}\right)$, where $l \geq 1$ and $b_{1}, \ldots, b_{l} \in \mathfrak{G}$; the number $l$ is called the length of the block and will be denoted by $l(\beta)$. A block $\beta=\left(b_{1}, \ldots, b_{l}\right)$ is said to occur in a sequence $\left\{a_{n}\right\}_{n=0}^{\infty}$ in $\mathfrak{G}$, starting at $k$ if $a_{k}=b_{1}, a_{k+1}=b_{2}, \ldots, a_{k+l-1}=b_{l}$. 
Definition 5.7. Let $\beta=\left(b_{1}, \ldots, b_{l}\right)$ be a block of Gaussian integers, with length $l \geq 2$. We say that $\beta$ is exceptional if $\left|b_{l}\right|=\sqrt{2}, b_{l-j}=2 \sigma^{j}\left(b_{l}\right)$ for $j=1, \ldots, l-2$, $b_{1} \neq 2 \sigma^{l-1}\left(b_{l}\right)$ and $\left|b_{1}-\sigma^{l-1}\left(b_{l}\right)\right|<2$.

We may bear in mind that the condition $\left|b_{l}\right|=\sqrt{2}$ means that $b_{l}$ is one of the four Gaussian integers, $1+i,-1+i,-1-i$ or $1-i$. For an exceptional block $\beta=\left(b_{1}, \ldots, b_{l}\right)$, the entries $b_{2}, \ldots, b_{l}$ are determined by $b_{l}$, and $b_{1}$ satisfies the condition $\left|b_{1}-b_{l}\right|<2$ if $l$ is odd and $\left.\mid b_{1}+\bar{b}_{l}\right) \mid<2$ if $l$ is even. For each of the four choices of $b_{l}$ as above, there are 8 possible choices for $b_{1}$ for which the condition holds, out of which 5 are such that $\left|b_{1}\right|>1$; we shall be interested in blocks for which the latter condition holds. We note that in particular if $b_{l}=1+i$ and $l$ is even, the 5 possible choices are $2 i,-1+i,-1+2 i,-2$ and $-2+i$. For other values of $b_{l}$ as above the corresponding possibilities for $b_{1}$ can be written either through direct computation or by using the above particular case and considerations of symmetry under the maps $z \mapsto-z$ and $z \mapsto \bar{z}$. Thus for each fixed $l$ there are only finitely many exceptional blocks. However, as the length can be arbitrary, the set of exceptional blocks is infinite.

Remark 5.8. If $\left\{a_{n}\right\}_{n=0}^{\infty}$ is the continued fraction expansion of $z \in \mathbb{C}^{\prime}$ with respect to the Hurwitz algorithm, then no exceptional block occurs in $\left\{a_{n}\right\}_{n=0}^{\infty}$, starting at $k \geq 1$; this was observed by Hurwitz [10] and is basic to the study in [10]; for an idea of the proof see the proof of Proposition 6.10 below. This observation motivates the definition as above (including with regard to the order of indices involved, which a priori may seem rather odd).

We now introduce two conditions on sequences of Gaussian integers.

Definition 5.9. Let $\left\{a_{n}\right\}_{n=0}^{\infty}$ be a sequence in $\mathfrak{G}$ with $\left|a_{n}\right|>1$ for all $n \geq 1$.

i) $\left\{a_{n}\right\}_{n=0}^{\infty}$ is said to satisfy Condition $(\mathrm{H})$ if no exceptional block of Gaussian integers occurs in $\left\{a_{n}\right\}_{n=0}^{\infty}$, starting at $k \geq 1$.

ii) $\left\{a_{n}\right\}_{n=0}^{\infty}$ is said to satisfy Condition $\left(\mathrm{H}^{\prime}\right)$ if no block $\left(b_{1}, b_{2}\right)$ (of length two) with $\left|b_{1}+\bar{b}_{2}\right|<2$ and $\left|b_{2}\right|=\sqrt{2}$ occurs in $\left\{a_{n}\right\}_{n=0}^{\infty}$, starting at $k \geq 1$.

Remark 5.10. We note that if Condition $\left(\mathrm{H}^{\prime}\right)$ holds, then Condition $(\mathrm{H})$ also holds, trivially, since for any exceptional block $\left(b_{1}, \ldots, b_{l}\right)$ we have $\left|b_{l}\right|=\sqrt{2}$ and $\mid b_{l-1}+$ $\bar{b}_{l} \mid<2$. It may be observed that when $a_{n+1}=1+i$, under Condition (H) $a_{n}$ cannot be one of $2 i,-1+i,-1+2 i,-2$ and $-2+i$, but can be $-2+2 i$, while under Condition $\left(\mathrm{H}^{\prime}\right) a_{n}$ is not allowed to be any of these elements. While Condition $\left(\mathrm{H}^{\prime}\right)$ is simpler and more convenient in some ways, unlike Condition $(\mathrm{H})$ it does not hold for the sequences corresponding to Hurwitz continued fraction expansions of certain $z \in \mathbb{C}^{\prime}$; in fact all blocks of the form $\left(b_{1}, \ldots, b_{l}\right)$ with $\left|b_{l}\right|=\sqrt{2}$ and $\left|b_{k}\right|=$ $2 \sigma^{l-k}\left(b_{l}\right)$ for all $k=1, \ldots, l-1$ can be seen to occur in the Hurwitz expansions. We shall however show that there exist algorithms for which Condition $\left(\mathrm{H}^{\prime}\right)$ holds (see Theorem 6.11).

Proposition 5.11. Let $\left\{a_{n}\right\}_{n=0}^{\infty}$ be a sequence in $\mathfrak{G}$, with $\left|a_{n}\right|>1$ for all $n \geq 1$, satisfying Condition (H), and let $\left\{p_{n}\right\},\left\{q_{n}\right\}$ be the corresponding $\mathcal{Q}$-pair. Then $\left|q_{n}\right|>\left|q_{n-1}\right|$ for all $n \geq 1$.

Proof. If possible suppose $\left|q_{n} / q_{n-1}\right| \leq 1$ for some $n \in \mathbb{N}$ and let $m$ be the smallest integer for which this holds. For all $0 \leq k \leq m$ let $z_{k}=q_{m-k} / q_{m-k-1}$. We have 
$\left|z_{0}\right| \leq 1$, and $\left|z_{k}\right|>1$ for all $k=1, \ldots, m$. Also

$$
z_{k}=\frac{q_{m-k}}{q_{m-k-1}}=a_{m-k}+\frac{q_{m-k-2}}{q_{m-k-1}}=a_{m-k}+z_{k+1}^{-1}=b_{k}+z_{k+1}^{-1}
$$

for $k=0, \ldots, m-1$, where $b_{k}=a_{m-k}$. We have $b_{k} \in \mathfrak{G}$ for $k=0, \ldots, m-1$ and $\left|b_{0}\right|>1$. We now apply Lemma 5.6. Then we have $\left|a_{m}\right|=\left|b_{0}\right|=\sqrt{2}$, and there exists $1 \leq k \leq m-1$ such that $b_{k} \neq 2 \sigma^{k}\left(b_{0}\right)$ and $\left|b_{k}-\sigma^{k}\left(b_{0}\right)\right|<2$ and $k$ is the smallest such integer, then it follows that $\left(a_{m-k}, \ldots, a_{m}\right)$ is an exceptional block. Since $\left\{a_{n}\right\}_{n=0}^{\infty}$ satisfies Condition (H) this is not possible. By the lemma we therefore get, in particular, that $b_{m-1}=2 \sigma^{m-1}\left(b_{0}\right)$ and $z_{m} \in \bar{B}\left(\sigma^{m}\left(b_{0}\right)\right)$. Then $\left|b_{m-1}\right|=2 \sqrt{2}$ and $\left|z_{m}\right| \leq \sqrt{2}+1$. But this is not possible since $z_{m}=q_{1} / q_{0}=a_{1}=$ $b_{m-1}$. The contradiction shows that $\left|q_{n}\right|>\left|q_{n-1}\right|$ for all $n \geq 1$. This proves the proposition.

Definition 5.12. A sequence $\left\{a_{n}\right\}$ in $\mathfrak{G}$ such that $\left|a_{n}\right|>1$ for all $n \geq 1$, is said to satisfy Condition (A) if for all $n \geq 1$ such that $\left|a_{n}\right| \leq 2$, Re $a_{n} a_{n+1} \geq \chi$, where $\chi=2$ if $\left|a_{n+1}\right|=\sqrt{2}$ and 0 otherwise. (If $\left|a_{n}\right| \leq 2$, then $a_{n+1}$ is required to be such that Re $a_{n} a_{n+1} \geq 0$; if moreover $\left|a_{n+1}\right|=\sqrt{2}$, then the condition further stipulates that Re $a_{n} a_{n+1} \geq 2$.)

Like Condition (H), Condition (A) also holds for the sequences $\left\{a_{n}\right\}$ arising from the Hurwitz algorithm (see [9]). Proposition [5.1] and Proposition [5.11 together imply the following.

Proposition 5.13. Let $\left\{a_{n}\right\}$ be a sequence in $\mathfrak{G}$, with $\left|a_{n}\right|>1$ for all $n \geq 1$, satisfying Conditions $(H)$ and $(A)$, and let $\left\{p_{n}\right\},\left\{q_{n}\right\}$ be the corresponding $\mathcal{Q}$-pair. Then $\left|q_{n}\right|>\left(\frac{\sqrt{5}+1}{2}\right)\left|q_{n-2}\right|$ for all $n \geq 2$.

An analogous conclusion with a smaller constant can also be proved under a weaker condition than Condition (A), which also has the advantage that it involves exclusion of occurrence of only finitely many pairs occuring as $\left(a_{n}, a_{n+1}\right)$.

Definition 5.14. A sequence $\left\{a_{n}\right\}$ in $\mathfrak{G}$ such that $\left|a_{n}\right|>1$ for all $n \geq 1$, is said to satisfy Condition ( $\left.\mathrm{A}^{\prime}\right)$ if for all $n \geq 1$ such that $\left|a_{n}\right| \leq 2$ and $\left|a_{n+1}\right| \leq 2$, either $\operatorname{Re} a_{n} a_{n+1}>0$ or $\operatorname{Im} a_{n} a_{n+1}= \pm 4$.

For $a_{n}, a_{n+1} \in \mathfrak{G}$ with $1<\left|a_{n}\right| \leq 2$ and $1<\left|a_{n+1}\right| \leq 2$, if the above condition holds, then $\left|a_{n} a_{n+1}+1\right|>\left|a_{n+1}\right|+(\sqrt{5}-1)$ (see the proof of Proposition 5.15 below), and on the other hand it can be verified that if $\left|a_{n} a_{n+1}+1\right|>\left|a_{n+1}\right|+1$, then the condition as in the definition holds.

Proposition 5.15. Let $\left\{a_{n}\right\}$ be a sequence in $\mathfrak{G}$ with $\left|a_{n}\right|>1$ for all $n \geq 1$, satisfying Conditions $(H)$ and $\left(A^{\prime}\right)$, and let $\left\{p_{n}\right\},\left\{q_{n}\right\}$ be the corresponding $\mathcal{Q}$ pair. Then $\left|q_{n}\right|>(\sqrt{5}-1)\left|q_{n-2}\right|$ for all $n \geq 1$.

Proof. Since $q_{-1}=0$ and $q_{1}=a_{1}$ the assertion trivially holds for $n=1$. Now suppose that $n \geq 2$. Since $\left\{a_{n}\right\}$ satisfies Condition (H), by Proposition $5.11\left\{\left|q_{n}\right|\right\}$ is increasing, and in particular $q_{n} \neq 0$ for all $n$. Recall that we have $q_{n}=a_{n} q_{n-1}+$ $q_{n-2}$ and hence $\left|q_{n} / q_{n-1}\right| \geq\left|a_{n}\right|-\left|q_{n-2} / q_{n-1}\right|>\left|a_{n}\right|-1$, as $\left|q_{n-2} / q_{n-1}\right|<1$. Since $\left|q_{n-1}\right|>\left|q_{n-2}\right|$, when $\left|a_{n}\right| \geq \sqrt{5}$, this yields $\left|q_{n} / q_{n-2}\right|>\left|q_{n} / q_{n-1}\right|>\sqrt{5}-1$, as desired. Similarly if $\left|a_{n-1}\right| \geq \sqrt{5}$, then we have $\left|q_{n} / q_{n-2}\right|>\left|q_{n-1} / q_{n-2}\right|>\sqrt{5}-1$. Now suppose that both $\left|a_{n}\right|$ and $\left|a_{n-1}\right|$ are less than $\sqrt{5}$, namely $\left|a_{n-1}\right| \leq 2$ and $\left|a_{n}\right| \leq 2$. We recall that $q_{n}=a_{n} q_{n-1}+q_{n-2}=a_{n}\left(a_{n-1} q_{n-2}+q_{n-3}\right)+q_{n-2}=$ 
$\left(a_{n} a_{n-1}+1\right) q_{n-2}+a_{n} q_{n-3}$, and hence $\left|q_{n} / q_{n-2}\right|>\left|a_{n} a_{n-1}+1\right|-\left|a_{n}\right|$. Let $a_{n} a_{n-1}=$ $x+y i, x, y \in \mathbb{Z}$. Since $\left|a_{n-1}\right| \leq 2$ and $\left|a_{n}\right| \leq 2$ it follows that $x$ and $y$ are even. If $|y|=4$, then $x=0$ and $\left|a_{n} a_{n-1}+1\right|-\left|a_{n}\right|=\sqrt{17}-2>\sqrt{5}-1$. Now suppose that $|y| \leq 2$. Then, since $\left\{a_{n}\right\}$ satisfies Condition $\left(\mathrm{A}^{\prime}\right), x=2$ or 4 . If $y=0$ and $x=2$, then $\left|a_{n}\right|=\sqrt{2}$ and we have $\left|a_{n} a_{n-1}+1\right|-\left|a_{n}\right|=3-\sqrt{2}>\sqrt{5}-1$. If $y=0$ and $x=4$, then $\left|a_{n} a_{n-1}+1\right|-\left|a_{n}\right|=5-2>\sqrt{5}-1$. Finally if $|y|=2$, then $\left|a_{n} a_{n-1}+1\right|-\left|a_{n}\right|=\sqrt{(x+1)^{2}+y^{2}}-2 \geq \sqrt{13}-2>\sqrt{5}-1$. This proves the proposition.

Remark 5.16. If $\left\{a_{n}\right\}$ is a sequence with $a_{n} \in \mathbb{Z} \subset \mathfrak{G}$ for all $n \geq 0$ and $\left|a_{n}\right| \geq 2$ for all $n \geq 1$, then Condition $(\mathrm{H})$ and Condition $\left(\mathrm{A}^{\prime}\right)$ are satisfied, and hence the conclusion of Proposition 5.15 holds for these sequences. If moreover $a_{n+1} / a_{n}$ is positive whenever $\left|a_{n}\right|=2$, then Condition (A) also holds and in this case the conclusion of Proposition 5.13 holds.

\section{Sequence Spaces and COntinued FRaCtion EXPAnsion}

In this section we introduce various spaces of sequences which serve as continued fraction expansions.

For $\theta>1$ let $\Omega_{E}^{(\theta)}$ denote the set of sequences $\left\{a_{n}\right\}$ such that if $\left\{p_{n}\right\},\left\{q_{n}\right\}$ is the corresponding $\mathcal{Q}$-pair, then, for all $n \geq 1,\left|q_{n}\right|>\left|q_{n-1}\right|$ and $\left|q_{n}\right| \geq \theta\left|q_{n-2}\right|$; we note that for these sequences $\left|q_{n}\right| \geq \theta^{(n-1) / 2}$ for all $n \geq 0$ (the suffix $E$ in the notation signifies exponential growth of the $\left|q_{n}\right|$ 's). By Proposition 5.13 the sequences $\left\{a_{n}\right\}$ satisfying Condition (H) and Condition (A) are contained in $\Omega_{E}^{\left(\frac{\sqrt{5}+1}{2}\right)}$; in particular, every sequence arising as the continued fraction expansion of any $z$ in $\mathbb{C}^{\prime}$ with respect to the Hurwitz algorithm is contained in $\Omega_{E}^{\left(\frac{\sqrt{5}+1}{2}\right)}$. Similarly by Proposition 5.15 the sequences $\left\{a_{n}\right\}$ satisfying Condition $(\mathrm{H})$ and Condition $\left(\mathrm{A}^{\prime}\right)$ are contained in $\Omega_{E}^{(\sqrt{5}-1)}$.

Theorem 6.1. Let $\left\{a_{n}\right\}_{n=0}^{\infty}$ be a sequence in $\Omega_{E}^{(\theta)}$, for some $\theta>1$, and let $\left\{p_{n}\right\},\left\{q_{n}\right\}$ be the corresponding $\mathcal{Q}$-pair. Then the following conditions hold:

(i) $\left\{p_{n} / q_{n}\right\}$ converges as $n \rightarrow \infty$;

(ii) if $z$ is the limit of $\left\{p_{n} / q_{n}\right\}$, then $\left|z-\frac{p_{n}}{q_{n}}\right| \leq \frac{c}{\left|q_{n}\right|^{2}}$, where $c=\frac{2 \theta^{2}}{\left(\theta^{2}-1\right)}$;

(iii) the limit $z$ is an irrational complex number, viz. $z \in \mathbb{C}^{\prime}$.

Proof. We have $p_{n+1} q_{n}-p_{n} q_{n+1}=(-1)^{n}$ for all $n \geq 0$, so $\frac{p_{n+1}}{q_{n+1}}-\frac{p_{n}}{q_{n}}=\frac{(-1)^{n}}{q_{n} q_{n+1}}$, and in turn $\left|\frac{p_{n+m}}{q_{n+m}}-\frac{p_{n}}{q_{n}}\right| \leq \sum_{k=0}^{m-1} \frac{1}{\left|q_{n+k} q_{n+k+1}\right|}$ for all $n \geq 0$ and $m \geq 1$. As $\left|q_{k} / q_{k-2}\right| \geq \theta$ for all $k \geq 1$ this shows that $\left\{p_{n} / q_{n}\right\}$ is convergent. This proves (i). Assertion (ii) then follows from Proposition 2.1. Finally, it is easy to see that such an approximation cannot hold for rational complex numbers, and hence the limit must be an irrational number.

For any $\left\{a_{n}\right\}$ in $\Omega_{E}^{(\theta)}, \theta>1$, the limit of $\left\{p_{n} / q_{n}\right\}$, where $\left\{p_{n}\right\},\left\{q_{n}\right\}$ is the corresponding $\mathcal{Q}$-pair, exists, and following the notation introduced earlier, in $\S 2$, we denote it by $\omega\left(\left\{a_{n}\right\}\right)$. The following corollary shows that for any fixed $\theta>1$ 
the convergents coming from possibly different representations of a number $z$ from $\Omega_{E}^{(\theta)}$ also converge to $z$, provided the corresponding indices go to infinity.

Corollary 6.2. Let $\theta>1, z \in \mathbb{C}^{\prime}$ be given, and $\left\{l_{j}\right\}$ be a sequence in $\mathbb{N}$ such that $l_{j} \rightarrow \infty$ as $j \rightarrow \infty$. Let $\left\{z_{j}\right\}$ be a sequence in $\mathbb{C}$ such that the following holds: for each $j$ there exists a sequence $\left\{a_{n}\right\}$ in $\Omega_{E}^{(\theta)}$ such that $z=\omega\left(\left\{a_{n}\right\}\right)$ and $z_{j}=p_{n} / q_{n}$ for some $n \geq l_{j}$, where $\left\{p_{n}\right\},\left\{q_{n}\right\}$ is the $\mathcal{Q}$-pair corresponding to $\left\{a_{n}\right\}$. Then $z_{j} \rightarrow z$ as $j \rightarrow \infty$.

Proof. By Theorem 6.1 we have $\left|z-z_{j}\right| \leq \frac{c}{\left|q_{n}\right|^{2}} \leq \frac{c}{\theta^{n-1}} \leq \frac{c}{\theta^{l_{j}-1}}$, where $c=$ $2 \theta^{2} /\left(\theta^{2}-1\right)$. As $l_{j} \rightarrow \infty$ this shows that $z_{j} \rightarrow z$, as $j \rightarrow \infty$.

We equip $\Omega_{E}^{(\theta)}, \theta>1$, with the topology induced as a subset of the space of all sequences $\left\{a_{n}\right\}$ with $a_{n} \in \mathfrak{G}$ and $\left|a_{n}\right|>1$ for all $n \geq 1$, the latter being equipped with the product topology as a product of the individual copies of $\mathfrak{G}$ (for the 0'th component) and $\mathfrak{G} \backslash\{0\}$ (for the later components) with the discrete topology.

Proposition 6.3. For all $\theta>1$ the map $\omega: \Omega_{E}^{(\theta)} \rightarrow \mathbb{C}$ is continuous.

Proof. Let $\left\{a_{n}\right\}$ be a sequence from $\Omega_{E}^{(\theta)}$ and let $\left\{p_{n}\right\},\left\{q_{n}\right\}$ be the corresponding $\mathcal{Q}$-pair. Let $\epsilon>0$ be given and $m \in \mathbb{N}$ be such that $\left|q_{m}\right|^{2}>2 c \epsilon^{-1}$, where $c=$ $2 \theta^{2} /\left(\theta^{2}-1\right)$. If $\left\{a_{n}^{\prime}\right\}$ is any sequence in $\Omega_{E}^{(\theta)}$ such that $a_{n}^{\prime}=a_{n}$ for $n=0,1, \ldots, m$, and $\left\{p_{n}^{\prime}\right\},\left\{q_{n}^{\prime}\right\}$ is the corresponding $\mathcal{Q}$-pair, then $p_{n}^{\prime}=p_{n}$ and $q_{n}^{\prime}=q_{n}$ for $n=$ $0,1, \ldots, m$. Then by Theorem 6.1 (ii), $\left|\omega\left(\left\{a_{n}\right\}\right)-\frac{p_{m}}{q_{m}}\right| \leq \frac{c}{\left|q_{m}\right|^{2}}<\epsilon / 2$, as well as $\left|\omega\left(\left\{a_{n}^{\prime}\right\}\right)-\frac{p_{m}}{q_{m}}\right|<\epsilon / 2$, so $\left|\omega\left(\left\{a_{n}^{\prime}\right\}\right)-\omega\left(\left\{a_{n}\right\}\right)\right|<\epsilon$. This shows that $\omega$ is continuous.

Remark 6.4. In general if $\left\{a_{n}\right\}_{n=0}^{\infty}$ is a sequence defining a continued fraction expansion of $z \in \mathbb{C}^{\prime}$, we may not have $\left|z-a_{0}\right| \leq 1$. For example, let $z^{\prime} \in B(0) \cap B(1+i) \cap \mathbb{C}^{\prime}$ and let $\left\{b_{n}\right\}$ be a continued fraction expansion of $z^{\prime}$ with $b_{0}=0$. Then for $z=\left(1+i-z^{\prime}\right)^{-1}$ we see that while $|z|>1$, the sequence $\left\{a_{n}\right\}$ with $a_{0}=0$, $a_{1}=1+i$ and $a_{n}=b_{n-1}$ for all $n \geq 2$ defines a continued fraction expansion of $z$, and we have $\left|z-a_{0}\right|>1$. This also means that when $\left\{a_{n}\right\}_{n=0}^{\infty}$ defines a continued fraction expansion of $z \in \mathbb{C}^{\prime}$, while for all $m \in \mathbb{N}$ there exists a (unique) $z_{m} \in \mathbb{C}^{\prime}$ such that the sequence $\left\{a_{n}\right\}_{n=m}^{\infty}$ defines a continued fraction expansion of $z_{m}$, the sequence $\left\{z_{m}\right\}$ may not be an iteration sequence of $z$, since the $z_{m}$ 's may not be in $\bar{B}(0,1)$. We next discuss a condition on the sequences $\left\{a_{n}\right\}_{n=0}^{\infty}$ which precludes such anomalies.

For a block $\beta=\left(b_{1}, \ldots, b_{l}\right)$ of Gaussian integers we call the block $\left(b_{l}, \ldots, b_{1}\right)$ the "opposite block" and denote it by $\widetilde{\beta}$. As with infinite sequences, given a finite sequence $\left\{a_{n}\right\}_{n=0}^{m}$ of Gaussian integers, we say that a block $\beta=\left(b_{1}, \ldots, b_{l}\right)$ occurs in $\left\{a_{n}\right\}_{n=0}^{m}$ starting at $k$, if $a_{k}=b_{1}, a_{k+1}=b_{2}, \ldots, a_{k+l-1}=b_{l}$. The following lemma involves a condition on the opposite blocks, to those occurring in the given sequence, not being exceptional (see Definition [5.7).

Lemma 6.5. Let $\left\{a_{n}\right\}_{n=0}^{m}$ be a finite sequence with $a_{n} \in \mathfrak{G}$ for all $n \geq 0$ and $\left|a_{n}\right|>1$ for $n=1, \ldots, m$. Let $\left\{p_{n}\right\}_{n=0}^{m}$ and $\left\{q_{n}\right\}_{n=0}^{m}$ be the corresponding $\mathcal{Q}$-pair. Suppose that for any block $\beta$ occurring in $\left\{a_{n}\right\}_{n=0}^{m}$ starting at $k$, where $k \geq 1$, the opposite block $\widetilde{\beta}$ is not an exceptional block. Then $\left|\frac{p_{m}}{q_{m}}-a_{0}\right|<1$. 
Proof. Let $\zeta=p_{m} / q_{m}$ and let $\zeta_{0}, \zeta_{1}, \ldots, \zeta_{m}$ be defined by $\zeta_{0}=\zeta$, and $\zeta_{n}=\left(\zeta_{n-1}-\right.$ $\left.a_{n-1}\right)^{-1}$ for $n=1, \ldots, m$. Then we see that $\zeta_{m}=a_{m}$. Suppose that $\left|\zeta-a_{0}\right| \geq 1$. Then we have $\left|\zeta_{m}\right|=\left|a_{m}\right|>1$, while on the other hand $\left|\zeta_{1}\right|=\left|\zeta-a_{0}\right|^{-1} \leq 1$. Hence there exists $1 \leq k \leq m-1$ such that $\left|\zeta_{k}\right| \leq 1$ and $\left|\zeta_{k+n}\right|>1$ for $n=1, \ldots, m-k$. For all $0 \leq n \leq m-k$ let $z_{n}=\zeta_{k+n}$ and $b_{n}=a_{n+k}$. Then we have $\left|z_{0}\right| \leq 1$ and $\left|z_{n}\right|>1$ for $n=1, \ldots, m-k$. Also $z_{n}-z_{n+1}^{-1}=\zeta_{n+k}-\zeta_{n+k+1}^{-1}=a_{n+k}=b_{n}$, for $0 \leq n \leq m-k-1$. By the condition in the hypothesis, for any $n$ the block $\left(b_{n}, \ldots, b_{0}\right)$ is not exceptional, which means that if $\left|b_{0}\right|=\sqrt{2}$, then for $1 \leq n \leq$ $m-k-1$, either $\left|b_{n}-\sigma^{n}\left(b_{0}\right)\right| \geq 2$ or $b_{n}=2 \sigma^{n}\left(b_{0}\right)$. Hence by Lemma 5.6 we have $\left|b_{0}\right|=\sqrt{2},\left|z_{m-k}\right| \leq \sqrt{2}+1$ and $\left|b_{m-k}\right|=2 \sqrt{2}$. But this is a contradiction since $b_{m-k}=a_{m}=\zeta_{m}=z_{m-k}$. Therefore $\left|\frac{p_{m}}{q_{m}}-a_{0}\right|<1$.

In light of the lemma we introduce the following definition; note that it differs from Condition $(\mathrm{H})$, with regard to the pairs to which it applies, they being opposite in the two conditions.

Definition 6.6. We say that a sequence $\left\{a_{n}\right\}_{n=0}^{\infty}$ satisfies Condition $\left(\mathrm{H}^{*}\right)$ if for every $n \geq 1$ such that $\left|a_{n}\right|=\sqrt{2}$, we have $\left|a_{n}+\bar{a}_{n+1}\right| \geq 2$.

We note that if $\left\{a_{n}\right\}_{n=0}^{\infty}$ is a sequence satisfying Condition $\left(\mathrm{H}^{*}\right)$, then for any block $\beta$ occurring in $\left\{a_{n}\right\}_{n=0}^{\infty}$ the block $\tilde{\beta}$ is not exceptional. Lemma 6.5 therefore implies the following.

Corollary 6.7. Let $\left\{a_{n}\right\}_{n=0}^{\infty}$ be a sequence with $\left|a_{n}\right|>1$ for all $n \geq 1$, satisfying Condition $\left(H^{*}\right)$, and suppose that for the corresponding $\mathcal{Q}$-pair $\left\{p_{n}\right\},\left\{q_{n}\right\}$ the sequence $\left\{p_{n} / q_{n}\right\}$ converges, say $p_{n} / q_{n} \rightarrow z \in \mathbb{C}$ as $n \rightarrow \infty$. Then $\left|z-a_{0}\right| \leq 1$. Furthermore, if for all $m \geq 1$ the sequence $\left\{p_{n}^{(m)} / q_{n}^{(m)}\right\}$, where $\left\{p_{n}^{(m)}\right\},\left\{q_{n}^{(m)}\right\}$ is the $\mathcal{Q}$-pair corresponding to $\left\{a_{n}\right\}_{n=m}^{\infty}$, converges, say $p_{n}^{(m)} / q_{n}^{(m)} \rightarrow z_{m}$, then $\left\{z_{m}\right\}$ is an iteration sequence for $z$.

Proof. Under the condition as in the hypothesis, by Lemma6.5 we get that $\mid p_{n} / q_{n}-$ $a_{0} \mid<1$ for all $n \geq 1$. Since $p_{n} / q_{n} \rightarrow z$ we get that $\left|z-a_{0}\right| \leq 1$. Clearly $z_{1}=(z-a)^{-1}$ and the preceding assertion implies that $\left|z_{1}\right| \geq 1$, and in turn we also get that $\left|z_{1}-a_{1}\right|<1$. Repeating the argument we see that $\left|z_{n}\right| \geq 1$ for all $n$. Since $z_{n}-z_{n+1}^{-1}=a_{n}$ for all $n \geq 0$ this shows that $\left\{z_{m}\right\}$ is an iteration sequence for $z$.

The spaces $\Omega_{E}^{(\theta)}$ are defined in terms of the growth of the $\left|q_{n}\right|$ 's, and do not seem to be amenable to a priori description, though of course as seen earlier verification of certain conditions on the sequences enables us to conclude that they belong to $\Omega_{E}^{(\theta)}$ for certain values of $\theta$. Our next objective is to describe a space of sequences defined in terms of non-occurrence of certain simple blocks, related to Conditions $\left(\mathrm{H}^{\prime}\right),\left(\mathrm{H}^{*}\right)$ and $\left(\mathrm{A}^{\prime}\right)$, which yield continued fraction expansions. We begin by introducing an algorithm such that the corresponding sequences of partial quotients satisfy these conditions.

Let $S$ be the square $\left\{z=x+i y|| x\left|\leq \frac{1}{2},\right| y \mid \leq \frac{1}{2}\right\}$. Let $P$ be the set of 8 points on the boundary of $S$ such that one of the coordinates is $\pm \frac{1}{6}$ (the other being $\pm \frac{1}{2}$ ). Let $R$ be the set defined by

$$
R=\left\{z \in S|| z, p \mid \geq \frac{1}{3} \text { for all } p \in P\right\} .
$$


It may be noted that $R$ is a star-like subset with the origin as the center, bounded by 8 circular segments; see Figure 1 . Let $C$ denote the set of four corner points of $S$, namely $\frac{1}{2}( \pm 1 \pm i)$. The set $C+R$ consists of the union of four translates of $R$ to the four corners of $S$.

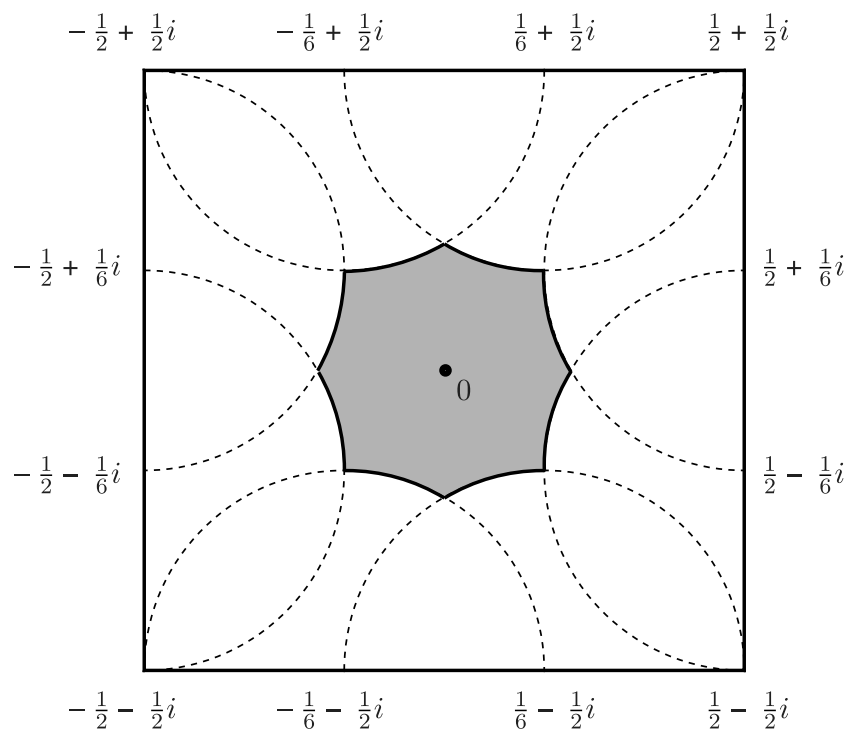

FiguRE 1. The set $R$.

We now define a choice function for a continued fraction algorithm as follows: let $z \in \mathbb{C}^{*}$ and $z_{0} \in \mathfrak{G}$ be such that $z-z_{0} \in S$. If $z-z_{0} \notin C+R$ we define $f(z)$ to be $z_{0}$, and if $z-z_{0} \in C+R$ we choose $f(z)$ to be the odd Gaussian integer nearest to $z$ (recall that a Gaussian integer $z=x+i y, x, y \in \mathbb{Z}$, is said to be odd if $x+y$ is odd). The choice for $z_{0}$ as above may not be unique, but the condition $z-z_{0} \notin C+R$ does not depend on the specific choice. The choices of $z_{0}$ and the nearest odd integer are not unique for certain $z$, but we shall assume that choosing the "nearest" is done by some convention - this will not play any role in the sequel. We shall call the algorithm corresponding to the function $f$ as above the PPOI algorithm, using the acronym for "partially preferring odd integers" as the name.

For each $c \in C$ let $T(c)$ denote the closed triangular subset of $S$ bounded by the three vertices of $S$ other than $c$. Also for each $c \in C$ let $\Psi(c)=c+(R \cap T(c))=$ $\{c+z \mid z \in R \cap T(c)\}$ and let $\Psi(C)=\bigcup_{c \in C} \Psi(c)$.

Remark 6.8. Let $z \in C^{*}$ and $z_{0}$ and $f(z)$ be as above. If $z-z_{0} \notin C+R$, then $z-f(z)=z-z_{0} \in S \backslash(C+R)=S \backslash \Psi(C)$. Now suppose $z-z_{0} \in C+R=\Psi(C)$. If $z_{0}$ is odd, then $z-f(z)=z-z_{0} \in S \cap \Psi(C)$. Lastly suppose $z_{0}$ is even. For definiteness suppose that $z-z_{0} \in \Psi\left(\frac{1}{2}(1+i)\right)$ (the other cases may be dealt with similarly). Then $f(z)$ is either $z_{0}+1$ or $z_{0}+i$. Hence $z-f(z)=\left(z-z_{0}\right)-1$ or $\left(z-z_{0}\right)-i$. Since $z-z_{0} \in \Psi\left(\frac{1}{2}(1+i)\right)$ we see that $\left(z-z_{0}\right)-1 \in \Psi\left(\frac{1}{2}(-1+i)\right)$ and $\left(z-z_{0}\right)-i \in \Psi\left(\frac{1}{2}(1-i)\right)$. Thus in this last case $z-f(z)$ is contained in $\Psi(C) \backslash S$. Altogether we see that $z-f(z)$ is always contained in $S \cup \Psi(C)$, which means that the fundamental set $\Phi$ of the PPOI algorithm is contained in $S \cup \Psi(C)$. A perusal of the above argument also shows that $\Phi=S \cup \Psi(C)$. 


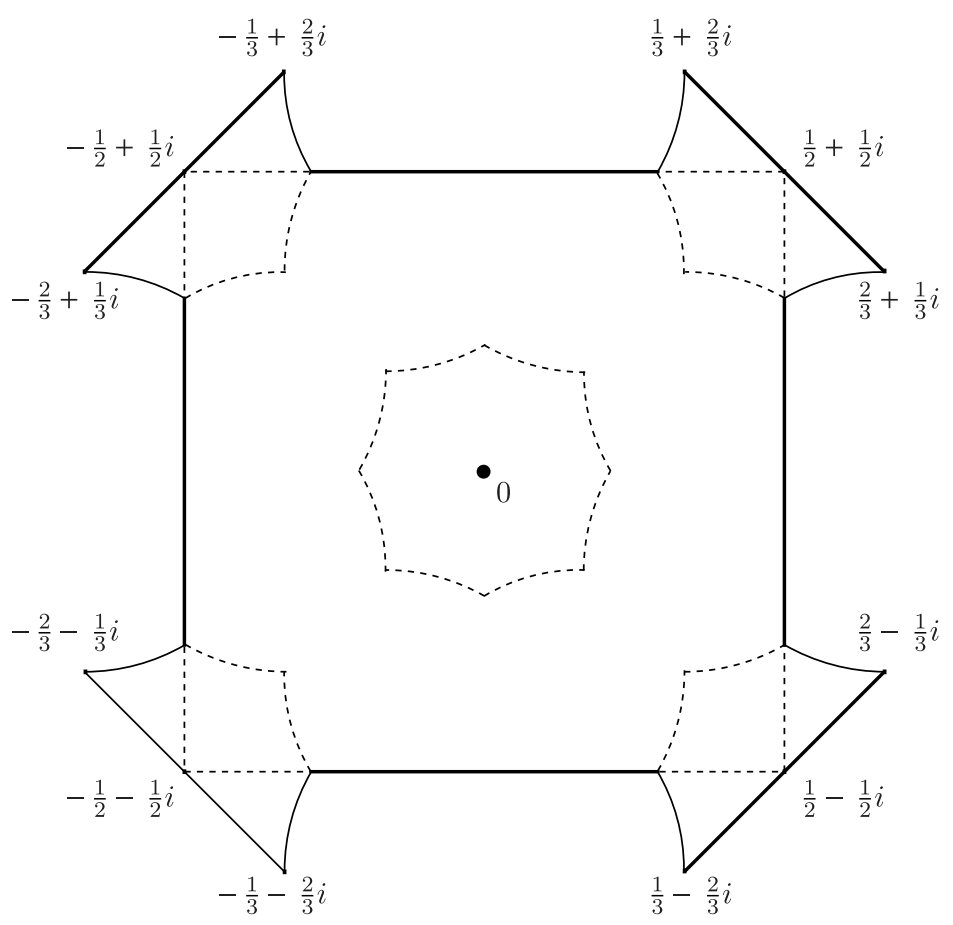

Figure 2. The fundamental set $\Phi$.

Remark 6.9. Let $z \in \mathbb{C}^{\prime},\left\{a_{n}\right\}$ be the associated sequence of partial quotients with respect to the PPOI algorithm, and $\left\{z_{n}\right\}$ be the corresponding iteration sequence. Consider $n \geq 1$ to be such that $a_{n}=1+i$. Then $z_{n} \in(1+i)+S$, and since $n \geq 1$, $z_{n}$ is also contained in $\Phi^{-1}$. Since $\Phi$ is contained in $\{z=x+i y \mid x-y \leq 1\}, \Phi^{-1}$ is in the complement of the ball $B\left(\frac{1}{2}(1+i), \frac{1}{\sqrt{2}}\right)$. Secondly, by a similar argument we see that $(\Phi \backslash S)^{-1}$ is disjoint from the balls $B(2)$ and $B(2 i)$, and on the other hand, so is $((1+i)+S) \cap S^{-1}$. Thus $z_{n} \notin B(2) \cup B(2 i)$. Therefore $z_{n+1}^{-1}=z_{n}-a_{n}$ is in the complement of the balls $B\left(-\frac{1}{2}(1+i), \frac{1}{\sqrt{2}}\right), B(1-i)$ and $B(-1+i)$. In turn we get that $z_{n+1}$ is contained in $\{z=x+i y \mid x-y \geq-1\}$ and is in the complement of $B(1+i)$ and $B(-1-i)$.

Proposition 6.10. Let $z \in \mathbb{C}^{\prime}$ and $\left\{a_{n}\right\}$ be the associated sequence of partial quotients with respect to the PPOI algorithm. Then $\left\{a_{n}\right\}$ satisfies Condition $\left(H^{\prime}\right)$, Condition $\left(H^{*}\right)$ and Condition $\left(A^{\prime}\right)$.

Proof. Let us first consider Condition $\left(\mathrm{H}^{*}\right)$. We have to show that for $n \geq 1$, if $\left|a_{n}\right|=\sqrt{2}$, then $\left|a_{n}+\bar{a}_{n+1}\right| \geq 2$. Due to the symmetries with respect to $z \mapsto-z$ and $z \mapsto \bar{z}$, it suffices to consider the case with $a_{n}=1+i$. From Remark 6.9 we see that in this case $a_{n+1}$ does not belong to the set $\{-2,-1+i, 2 i,-2+i,-1+2 i,-2+2 i\}$, which is precisely the set of Gaussian integers $a$ such that $|a|>1$ and $|1+i+\bar{a}|<2$. This shows that Condition $\left(\mathrm{H}^{*}\right)$ holds.

We shall next show that if $\mid a_{n+1}=\sqrt{2}$, then $\left|\bar{a}_{n}+a_{n+1}\right| \geq 2$, and if furthermore $\left|a_{n}\right| \leq 2$ then $\operatorname{Re} a_{n} a_{n+1}>0$; this will verify Condition $\left(\mathrm{H}^{\prime}\right)$ and part of Condition $\left(\mathrm{A}^{\prime}\right)$. By symmetry considerations it suffices to prove this when 
$a_{n+1}=1+i$, and in this case we have to prove that $a_{n}$ does not belong to the sets $\{-2,-1+i, 2 i,-2+i,-1+2 i,-2+2 i\}$ and $\{-1+i,-1-i, 1+i,-2,2 i\}$.

Let $\left\{z_{n}\right\}$ denote the iteration sequence corresponding to $z$, with respect to the PPOI algorithm. Since $1+i$ is even this means that $z_{n+1}$ is contained in $(1+$ $i)+(S \backslash \Psi(C))$ (see Remark 6.8). We note that for $z \in(1+i)+S$, if $z^{-1} \in S$, then $z^{-1} \in \Psi\left(\frac{1}{2}(1-i)\right)$. Since $z_{n}-a_{n}=z_{n+1}^{-1} \in(1+i)+S$, this shows that if $z_{n}-a_{n} \in S$, then $z_{n}-a_{n} \in \Psi\left(\frac{1}{2}(1-i)\right)$. On the other hand, if $z_{n}-a_{n} \notin S$, since it is contained in $\Phi$ and also the fourth quadrant, we get that $z_{n}-a_{n} \in \Psi\left(\frac{1}{2}(1-i)\right)$, in this case as well. Since $f^{-1}(a)$ is disjoint from $\Phi(c)$ for all $c \in C$ when $a$ is even, this implies, first, that $a_{n}$ is odd. Hence $a_{n}$ does not take values from the sets as above, except possibly $-2+i$ or $-1+2 i$. Now suppose $a_{n}=-1+2 i$. Recalling that $z_{n}-a_{n} \in \Psi\left(\frac{1}{2}(1-i)\right)$, we get $z_{n} \in(-1+2 i)+\Psi\left(\frac{1}{2}(1-i)\right)$. As $n \geq 1$ and $z_{n}=\left(z_{n-1}-a_{n-1}\right)^{-1}, z_{n}$ is also contained in $\Phi^{-1}=S^{-1} \cup(\Psi(C))^{-1}$. We observe that $(-1+2 i)+\Psi\left(\frac{1}{2}(1-i)\right)$ is contained in $B(i)$; the distance of the vertices of the former set from $i$ are the same as the distances of the vertices of $R$ from the corner points, viz. points of $C$, and these may be seen to be (strictly) less than 1. Therefore $(-1+2 i)+\Psi\left(\frac{1}{2}(1-i)\right)$ is disjoint from $S^{-1}$. Hence we get that $z_{n} \in(\Psi(C))^{-1}$. For $c \neq \frac{1}{2}(-1-i), \Psi(c)^{-1}$ is disjoint from the second quadrant. Hence $z_{n}$ must be contained in $\left(\Psi\left(\frac{1}{2}(-1-i)\right)\right)^{-1}$. We see that $\Psi\left(\frac{1}{2}(-1-i)\right)$ is disjoint from the $B\left(-\frac{2 i}{3}, \frac{1}{3}\right)$ and the set of inverses of the latter consists of $B(2 i)$. But $(-1+2 i)+\Psi\left(\frac{1}{2}(1-i)\right)$ can be seen to be contained in $B(2 i)$. Therefore it is disjoint from $\left(\Psi\left(\frac{1}{2}(-1-i)\right)\right)^{-1}$ as well. This is a contradiction, showing that $a_{n} \neq-1+2 i$. By a similar argument (or by symmetry) we see that $a_{n} \neq-2+i$. This verifies that if $a_{n+1}=1+i$, then $a_{n}$ is not contained in the sets as above.

To complete the proof it remains to show that if $\left|a_{n}\right| \leq 2$ and $\left|a_{n+1}\right|=2$, then either $\operatorname{Re} a_{n} a_{n+1}>0$ or $\operatorname{Im} a_{n} a_{n+1}= \pm 4$. By symmetry considerations it suffices to prove this in the cases $a_{n+1}=2$ or $2 i$. When $a_{n+1}=2$ we have to show that $a_{n}$ is not contained in $\{-1+i,-1-i,-2\}$ and when $a_{n+1}=2 i$ we have to show that $a_{n}$ is not contained in $\{-1+i, 1+i, 2 i\}$.

Now suppose $a_{n+1}=2$. Then we have $z_{n+1} \in(2+S \backslash \Psi(C)) \cap \Phi^{-1} \subset(2+S)$. We note that the set of inverses of $B\left(\frac{1}{2}(1-i), \frac{1}{\sqrt{2}}\right)$ is the affine half-plane cut off by the line joining $i$ and 1 and containing $2+S$. Therefore $z_{n+1}^{-1}=z_{n}-a_{n}$ is contained in $B\left(\frac{1}{2}(1-i), \frac{1}{\sqrt{2}}\right)$. Similarly we see that it is also contained in $B\left(\frac{1}{2}(1+i), \frac{1}{\sqrt{2}}\right)$. Now suppose $a_{n}=-1+i$. Then $z_{n} \in(-1+i+S) \cap \Phi^{-1}$, and the latter is seen to be contained in $(-1+i+S) \backslash B\left(\frac{1}{2}(-1+i), \frac{1}{\sqrt{2}}\right)$. This however implies that $z_{n}-a_{n}$ is not contained in $B\left(\frac{1}{2}(1-i), \frac{1}{\sqrt{2}}\right)$, contradicting what we noted above. This shows that $a_{n} \neq-1+i$, and by a similar argument, using the other observation made above, we conclude that $a_{n} \neq-1-i$. Also, noting that $z_{n+1}^{-1}$ is contained in $B(1)$ while $(-2+S) \cap \Phi^{-1}$ is disjoint from $-2+B(1)=B(-1)$, we conclude that $a_{n}$ is not -2 .

A similar argument also shows that if $a_{n+1}=2 i$, then $a_{n}$ is not one of $-1+i, 1+i$ and $2 i$. This completes the proof.

We can now conclude the following.

Theorem 6.11. Every irrational complex number $z$ admits a continued fraction expansion $\left\{a_{n}\right\}_{n=0}^{\infty}, a_{n} \in \mathfrak{G}$ for all $n$, satisfying the following conditions:

(i) $\left|a_{n}\right|>1$ for all $n \geq 1$; 
(ii) for $n \geq 1$, if either $\left|a_{n}\right|=\sqrt{2}$ or $\left|a_{n+1}\right|=\sqrt{2}$, then $\left|a_{n}+\bar{a}_{n+1}\right| \geq 2$;

(iii) for $n \geq 1$, if $\left|a_{n}\right| \leq 2$ and $\left|a_{n+1}\right| \leq 2$, then either $\operatorname{Re} a_{n} a_{n+1}>0$ or $\operatorname{Im} a_{n} a_{n+1}= \pm 4$.

Conversely, if $\left\{a_{n}\right\}_{n=0}^{\infty}, a_{n} \in \mathfrak{G}$, is a sequence satisfying conditions (i), (ii) and (iii), and $\left\{p_{n}\right\},\left\{q_{n}\right\}$ is the corresponding $\mathcal{Q}$-pair, then the following holds:

(a) $\left\{\left|q_{n}\right|\right\}$ is strictly monotonically increasing and $\left|q_{n}\right|>(\sqrt{5}-1)\left|q_{n-2}\right|$ for all $n \geq 1$;

(b) $\left\{p_{n} / q_{n}\right\}$ converges to an irrational complex number $z$ as $n \rightarrow \infty$, (so $\left\{a_{n}\right\}$ defines a continued fraction expansion of $z$ ), and $\left|z-a_{0}\right| \leq 1$;

(c) for $m \in \mathbb{N}$, if $\left\{p_{n}^{(m)}\right\},\left\{q_{n}^{(m)}\right\}$ is the $\mathcal{Q}$-pair corresponding to $\left\{a_{n+m}\right\}_{n=0}^{\infty}$, then $\left\{p_{n}^{(m)} / q_{n}^{(m)}\right\}$ converges to an irrational complex number $z_{m}$, and $\left\{z_{m}\right\}_{m=0}^{\infty}$ (with $z_{0}=z$ ) is an iteration sequence for $z$;

(d) $z$ is a quadratic surd if and only if $\left\{z_{n} \mid n \in \mathbb{N}\right\}$ is finite.

Proof. Existence of a continued fraction expansion satisfying conditions (i), (ii) and (iii) follows from Proposition 6.10. In the converse part, assertion (a) follows from Propositions 5.11 and 5.15. assertion (b) follows from Theorem 6.1 and Corollary 6.7, and the latter also implies that $\left\{z_{n}\right\}$ is an iteration sequence for $z$, thus proving (c). Assertion (d) follows from Proposition 4.1 and Corollary 4.3 together with assertion (c).

Remark 6.12. Let $\Omega_{F}$ be the class of sequences $\left\{a_{n}\right\}_{n=0}^{\infty}, a_{n} \in \mathfrak{G}$, for all $n$, satisfying Conditions (i), (ii) and (iii) in Theorem [6.11, namely such that $\left|a_{n}\right|>1$ and Conditions $\left(\mathrm{H}^{\prime}\right),\left(\mathrm{H}^{*}\right)$ and $\left(\mathrm{A}^{\prime}\right)$ hold. Let $\left(b, b^{\prime}\right)$ be a block of length 2 with $|b| \leq 2$ and $\left|b^{\prime}\right| \leq 2$ occurring in some sequence $\left\{a_{n}\right\}_{n=0}^{\infty}$ in $\Omega_{F}$ (starting at $n \geq 1$ ). Then it is straightforward to verify that $\left(b, b^{\prime}\right)$ is one of $(b, \bar{b}),(b, b+\bar{b}),(b+\bar{b}, b)$ for some $b$ with $|b|=\sqrt{2}$, or one of $\left(b, b^{\prime}\right)$ with $|b|=\left|b^{\prime}\right|=2$ and $b b^{\prime} \neq-4$. Of the 64 blocks $\left(b, b^{\prime}\right)$ with $|b| \leq 2$ and $\left|b^{\prime}\right| \leq 2$, these account for 24 blocks. The other 40 are not allowed to occur. Apart from these, the blocks $(b,-2 \bar{b})$ and $(-2 \bar{b}, b)$, with $|b|=\sqrt{2}$, are also not allowed to occur. This exhausts the list and thus there are altogether 48 blocks, each of length 2 , that are "forbidden"; any sequence $\left\{a_{n}\right\}_{n=0}^{\infty}$, with $a_{n} \in \mathfrak{G}$ and $\left|a_{n}\right|>1$ in which none of the forbidden blocks occur, starting at $n \geq 1$, belongs to $\Omega_{F}$.

\section{Associated fractional transformations}

As with the usual continued fractions we can associate with the complex continued fractions transformations of the projective space, the complex projective space in this instance.

We denote by $\mathbb{C}^{2}$ the two-dimensional complex vector space, viewed as the space of 2-rowed columns with complex number entries. We denote by $e_{1}$ and $e_{2}$ the vectors $\left(\begin{array}{l}1 \\ 0\end{array}\right)$ and $\left(\begin{array}{l}0 \\ 1\end{array}\right)$, in $\mathbb{C}^{2}$, respectively. For all $z \in \mathbb{C}$ we denote by $v_{z}$ the vector $z e_{1}+e_{2}$.

We denote by $\mathbb{P}$ the corresponding one-dimensional complex projective space, consisting of complex lines (one-dimensional vector subspaces) in $\mathbb{C}^{2}$ and by $\pi$ : $\mathbb{C} \rightarrow \mathbb{P}$ the map defined by setting, for all $z \in \mathbb{C}, \pi(z)$ to be the complex line containing $v_{z}$. Also, for $r=1,2$ we denote by $\bar{e}_{r}$ the complex line spanned by $e_{r}$.

We denote by $S L(2, \mathbb{C})$ the group of $2 \times 2$ matrices with complex entries, with determinant 1 . The natural linear action of $S L(2, \mathbb{C})$ on $\mathbb{C}^{2}$ induces an action of 
the group on $\mathbb{P}$. We shall be using this action freely in the sequel, with no further mention. We denote by $S L(2, \mathfrak{G})$ the subgroup of $S L(2, \mathbb{C})$ consisting of all those elements with entries in $\mathfrak{G}$.

When $\mathbb{C}$ is realized as a subset of $\mathbb{P}$ via the map $\pi$ as above, the subset $\mathbb{C}^{\prime}$ (as before, consisting of complex numbers which are not rational) is readily seen to be invariant under the action of $S L(2, \mathfrak{G})$; the action of the element $\left(\begin{array}{ll}p & q \\ r & s\end{array}\right) \in$ $S L(2, \mathfrak{G})$ on $\mathbb{C}^{\prime}$ is then given by $z \mapsto \frac{p z+q}{r z+s}$ for all $z \in \mathbb{C}^{\prime}$.

We denote by $\rho$ the matrix defined by

$$
\rho=\left(\begin{array}{cc}
0 & -1 \\
1 & 0
\end{array}\right)
$$

For any $g \in S L(2, \mathbb{C})$ we denote by ${ }^{t} g$ the transpose of the matrix $g$. It can be verified that for any $g \in S L(2, \mathbb{C}),{ }^{t} g \rho g=\rho$; this identity will be used crucially below. For any $a \in \mathfrak{G}$ we denote by $U_{a}$ and $L_{a}$ the matrices defined by

$$
U_{a}=\left(\begin{array}{cc}
1 & a \\
0 & 1
\end{array}\right) \text { and } L_{a}=\left(\begin{array}{cc}
1 & 0 \\
a & 1
\end{array}\right)
$$

For a block $\beta=\left(b_{1}, \ldots, b_{l}\right)$ of Gaussian integers we define

$$
g(\beta)=U_{b_{1}} L_{b_{2}} \cdots U_{b_{l-1}} L_{b_{l}} \text { or } U_{b_{1}} L_{b_{2}} \cdots L_{b_{l-1}} U_{b_{l}},
$$

according to whether $l$ is even or odd, respectively.

We now introduce some more nomenclature and notation concerning blocks of Gaussian integers. Given two blocks $\beta=\left(b_{1}, \ldots, b_{l}\right)$ and $\beta^{\prime}=\left(b_{1}^{\prime}, \ldots, b_{l^{\prime}}^{\prime}\right)$, we denote by $\beta \beta^{\prime}$ the block $\left(b_{1}, \ldots, b_{l}, b_{1}^{\prime}, \ldots, b_{l^{\prime}}^{\prime}\right)$. Let $\beta, \beta^{\prime}$ be two blocks with $l\left(\beta^{\prime}\right) \leq$ $l(\beta)$. Then $\beta^{\prime}$ is called an initial block of $\beta$ if $\beta=\beta^{\prime} \beta^{\prime \prime}$ for some block $\beta^{\prime \prime}$, and $\beta^{\prime}$ is called an end block of $\beta$ if $\beta=\beta^{\prime \prime} \beta^{\prime}$ for some block $\beta^{\prime \prime}$. For a block $\beta=$ $\left(b_{1}, \ldots, b_{l}\right), b_{1}$ and $b_{l}$ will be called the first and last entries respectively. For a block $\beta=\left(b_{1}, \ldots, b_{l}\right)$ we denote by $\tilde{\beta}$ the block $\left(b_{l}, \ldots, b_{1}\right)$, with the entries arranged in reverse order.

Remark 7.1. Let $\left\{a_{n}\right\}_{n=0}^{l-1}$ be a sequence with $a_{n} \in \mathfrak{G},\left|a_{n}\right|>1$ for $n \geq 1$, and let $\beta$ be the block $\left(a_{0}, a_{1}, \ldots, a_{l-1}\right)$. Let $\left\{p_{n}\right\},\left\{q_{n}\right\}$ be the $\mathcal{Q}$-pair corresponding to $\left\{a_{n}\right\}_{n=0}^{l-1}$. Then it is straightforward to verify, by induction, that for $r=1$ and 2 , $g(\beta)\left(e_{r}\right)=p_{m} e_{1}+q_{m} e_{2}$, where $m$ is the largest odd integer less than $l$ if $r=1$ and the largest even integer less than $l$ if $r=2$; see the proof of Proposition 3.1 in [7].

For $\theta>1$, a sequence $\left\{\beta_{j}\right\}$ of blocks of Gaussian integers is said to $\theta$-represent a number $z \in \mathbb{C}^{\prime}$ if $l\left(\beta_{j}\right) \rightarrow \infty$, and for each $i$ there exists a sequence $\left\{a_{n}\right\}$ (depending on $i)$ in $\Omega_{E}^{(\theta)}$ such that $\omega\left(\left\{a_{n}\right\}\right)=z$ and $\beta_{j}$ is an initial block of $\left\{a_{n}\right\}$.

The following two results are generalizations of the corresponding results for simple continued fractions proved in [7, Proposition 3.1 and Lemma 2.4, to complex continued fractions in the generality being considered here; it may be worthwhile for the reader to see Lemma 2.2 and Example 2.3 in [7] for a broader perspective on the issue.

Theorem 7.2. Let $\zeta \in \mathbb{C}^{\prime}$ and $\left\{\beta_{j}\right\}$ be a sequence of blocks of Gaussian integers $\theta$-representing $\zeta$. Then as $j \rightarrow \infty$,

$$
g\left(\beta_{j}\right)(\pi(z)) \rightarrow \pi(\zeta) \text { for all } z \text { with }|z|>1,
$$

uniformly over $\{z \in \mathbb{C}|| z \mid \geq 1+\epsilon\}$, for all $\epsilon>0$. 
Proof. It will suffice to prove the assertion in the theorem separately with all $l\left(\beta_{j}\right)$ being odd and all being even. We shall give the argument when all $l\left(\beta_{j}\right)$ are even; a similar argument works in the other case. Let $z \in \mathbb{C}$ with $|z|>1$ be given. For each $j$ let $h_{j}$ and $k_{j}$ be the largest odd and even integers less than $l\left(\beta_{j}\right)$, respectively; as $l\left(\beta_{j}\right)$ are even we have $h_{j}=l\left(\beta_{j}\right)-1$ and $k_{j}=h_{j}-1$ for all $i$. Then by Remark 7.1 we have

$$
g\left(\beta_{j}\right)(\pi(z))=\pi\left(\frac{z p_{h_{j}}+p_{k_{j}}}{z q_{h_{j}}+q_{k_{j}}}\right)=\pi\left(\frac{p_{h_{j}}}{q_{h_{j}}} \frac{z+\left(p_{k_{j}} / p_{h_{j}}\right)}{z+\left(q_{k_{j}} / q_{h_{j}}\right)}\right) .
$$

Since $\left\{\beta_{j}\right\}$ is a sequence of blocks $\theta$-representing $\zeta$, by Corollary 6.2 we get that $\left\{\frac{p_{h_{j}}}{q_{h_{j}}}\right\}$ and $\left\{\frac{p_{k_{j}}}{q_{k_{j}}}\right\}$ converge to $\zeta$. Since $\left\{a_{n}\right\}$ belongs to $\Omega_{E}^{(\theta)}$ and $k_{j}<h_{j}$ we have $\left|q_{k_{j}}\right|<\left|q_{h_{j}}\right|$ for all $j$. As $|z|>1$ this shows that $\left\{z+\left(q_{k_{j}} / q_{h_{j}}\right)\right\}$ is a bounded sequence, bounded away from 0; moreover, whenever a subsequence of $\left\{z+\left(q_{k_{j}} / q_{h_{j}}\right)\right\}$ converges (to a non-zero number), the corresponding subsequence of $\left\{z+\left(p_{k_{j}} / p_{h_{j}}\right)\right\}$ also converges to the same number, since $\frac{p_{k_{j}} / p_{h_{j}}}{q_{k_{j}} / q_{h_{j}}}=\frac{p_{k_{j}}}{q_{k_{j}}} \frac{q_{h_{j}}}{p_{h_{j}}} \rightarrow \zeta \cdot \zeta^{-1}=1$. Thus we get that $g\left(\beta_{j}\right)(\pi(z)) \rightarrow \pi(\zeta)$ as $j \rightarrow \infty$. Moreover, clearly, for any $\epsilon>0$ the convergence is uniform over $\{z \in \mathbb{C}|| z \mid \geq 1+\epsilon\}$.

The next result is the analogue of Lemma 2.4 of [7] (and can be proved following the argument from there almost verbatim) over the field of complex numbers as scalars in the present instance; we omit the details.

Proposition 7.3. Let $\left\{g_{j}\right\}$ be an unbounded sequence in $S L(2, \mathbb{C})$. Suppose that $\varphi, \psi$ are in $\mathbb{P}$ such that the sets $\left\{\xi \in \mathbb{P} \mid g_{j}(\xi) \rightarrow \varphi\right\}$ and $\left\{\xi \in \mathbb{P} \mid{ }^{t} g_{j}(\xi) \rightarrow \psi\right\}$ have at least two elements each, and that $g_{j}(\rho(\psi))$ converges. Then $g_{j}(\xi) \rightarrow \varphi$ for all $\xi \neq \rho(\psi)$.

From Lemma 6.5 and Remark 7.1 we get the following.

Corollary 7.4. Let $\left\{a_{n}\right\}$ be a sequence satisfying Condition (H), and let $\beta=$ $\left(b_{1}, \ldots, b_{l}\right)$ be a block of Gaussian integers, with $l(\beta)$ even, occurring in $\left\{a_{n}\right\}$, starting at $k \geq 0$. Let $z_{r} \in \mathbb{C}, r=1,2$, be the numbers defined by $\pi\left(z_{r}\right)={ }^{t} g(\beta)\left(\bar{e}_{r}\right)$. Then $\left|z_{r}-b_{l}\right|<1$.

The following is our main technical result enabling us to conclude values of certain binary quadratic forms at pairs of Gaussian integers to be dense in $C$; see Corollaries 8.7 and 8.9. The result generalizes Theorem 3.2 of [7] for the case at hand.

Proposition 7.5. Let $\left\{a_{n}\right\}$ be a sequence in $\Omega_{E}^{(\theta)}, \theta>1$, and let $z=\omega\left(\left\{a_{n}\right\}\right)$. Let $\alpha, \beta \in \mathbb{C}^{\prime}$ and $\left\{\alpha_{j}\right\}$ and $\left\{\beta_{j}\right\}$ be sequences of blocks of Gaussian integers $\theta$ representing $\alpha$ and $\beta$ respectively. Suppose that the following conditions are satisfied:

i) for all $j, \alpha_{j}$ and $\beta_{j}$ are of even length and their first entries have absolute value greater than 1 , and the last entry of $\alpha_{j}$ has absolute value greater than 2 ;

ii) there exists a sequence $\left\{s_{j}\right\}$ of even integers such that for all $i$ the block $\tilde{\alpha}_{j} \beta_{j}$ occurs in $\left\{a_{n}\right\}$ starting at $s_{j}$, and moreover $s_{i+1}>s_{j}+l\left(\alpha_{j}\right)+l\left(\beta_{j}\right),\left|a_{s_{j}-1}\right|>2$ and $\left|a_{s_{j}+l\left(\alpha_{j}\right)+l\left(\beta_{j}\right)}\right|>2$.

For all $j$ let $z_{j}=\left(a_{0}, \ldots, a_{s_{j}-1}\right)$ and $g_{j}=g\left(\alpha_{j}\right)^{t} g\left(z_{j}\right)$. Then, as $j \rightarrow \infty$,

$$
g_{j}(\pi(\zeta)) \rightarrow \pi(\alpha) \text { for all } \zeta \neq \rho(\pi(z)) \text {, and } g_{j}(\rho(\pi(z))) \rightarrow \rho(\pi(\beta)) .
$$


Proof. Let $j \in \mathbb{N}$, and for $r=1,2$ let $\zeta_{r}^{(j)}$ be defined by $\pi\left(\zeta_{r}^{(j)}\right)={ }^{t} g\left(z_{j}\right)\left(\bar{e}_{r}\right)$. Since $\left|a_{s_{j-1}}\right|>2$, and hence at least $\sqrt{5}$, by Corollary $\left[7.4\right.$ it follows that $\left|\zeta_{r}^{(j)}\right| \geq$ $\sqrt{5}-1>1$. Since this holds for all $i$ by Theorem 7.2 we get that, as $j \rightarrow \infty$, $g_{j}\left(\bar{e}_{r}\right)=g\left(\alpha_{j}\right)\left(\pi\left(\zeta_{r}^{(j)}\right)\right) \rightarrow \pi(\alpha)$, for $r=1$ and 2. Similarly, under the conditions in the hypothesis we get ${ }^{t} g_{j}\left(\bar{e}_{r}\right) \rightarrow \pi(z)$ for $r=1,2$.

We next show that $g_{j}(\rho(\pi(z))) \rightarrow \rho(\pi(\beta))$. Then by Proposition 7.3 this would imply the conclusion as stated in the present proposition. Let $1 \leq h<j$. Then from the conditions in the hypothesis we see that $z_{j}$ may be written as $z_{j}=z_{j} \tilde{\alpha}_{j} \beta_{j} \varphi_{h, j}$, where $\varphi_{h, j}$ is the end-block of $z_{j}$ of length $s_{h+1}-s_{j}-l\left(\alpha_{j}\right)-l\left(\beta_{j}\right)>0$. Therefore $g\left(z_{j}\right)=g\left(z_{j}\right)^{t} g\left(\alpha_{j}\right) g\left(\beta_{j}\right) g\left(\varphi_{h, j}\right)$, and hence

$$
g_{j} \rho g\left(z_{j}\right)=g\left(\alpha_{j}\right)^{t} g\left(z_{j}\right) \rho g\left(z_{j}\right)^{t} g\left(\alpha_{j}\right) g\left(\beta_{j}\right) g\left(\varphi_{h, j}\right)=\rho g\left(\beta_{j}\right) g\left(\varphi_{h, j}\right),
$$

using (twice) the fact that ${ }^{t} g \rho g=\rho$ for all $g \in S L(2, \mathbb{C})$. We note that $s_{j}+l\left(\alpha_{j}\right)+$ $l\left(\beta_{j}\right)$ are even and $a_{s_{j}+l\left(\alpha_{j}\right)+l\left(\beta_{j}\right)}$ is the first entry of $\varphi_{h, j}$. Since $\left|a_{s_{j}+l\left(\alpha_{j}\right)+l\left(\beta_{j}\right)}\right| \geq$ $\sqrt{5}$, arguing as in the first part we conclude that $\rho g\left(\beta_{j}\right) g\left(\varphi_{h, j}\right)\left(\bar{e}_{r}\right)$ converges to $\rho(\pi(\beta))$, as $h$ and $j$ tend to infinity, for $r=1,2$. Hence $g_{j} \rho g\left(z_{j}\right)\left(\pi\left(e_{r}\right)\right), r=1,2$, converge to $\rho(\pi(\beta))$, as $h$ and $j$ tend to infinity. Since any limit point of the sequence $\left\{g_{j}(\rho(\pi(z)))\right\}$ is a limit point of $g_{j} \rho g\left(z_{j}\right)\left(e_{r}\right)$ as $h$ and $j$ tend to $\infty$, this shows that $g_{j}(\rho(\pi(z))) \rightarrow \rho(\pi(\beta))$. This completes the proof of the proposition.

\section{Application to Values of quadratic Forms}

In this section we apply our results on complex continued fractions to the study of values of quadratic forms with complex coefficients over the set of pairs of Gaussian integers. It was proved by G. A. Margulis in response to a long standing conjecture due to A. Oppenheim that for any non-degenerate indefinite real quadratic form $Q$ in $n \geq 3$ variables which is not a scalar multiple of a rational quadratic form, the set of values of $Q$ on the set of $n$-tuples of integers forms a dense subset of $\mathbb{R}$. This was generalized by A. Borel and G. Prasad to quadratic forms over other locally compact fields [5], and in particular it is known that for a non-degenerate complex quadratic form in $n \geq 3$ variables which is not a scalar multiple of a rational form, the set of values on $n$-tuples of Gaussian integers is dense in $\mathbb{C}$. The reader is referred to [6] and $[12$ for a discussion on the overall theme, including Ratner's pioneering work on dynamics of unipotent flows on homogeneous spaces to which the results are related. The case of binary quadratic forms, viz. $n=2$, does not however fall in the ambit of the theme, and in fact the corresponding statement is not true for all irrational indefinite binary quadratic forms. For example, if $\lambda$ is a badly approximable number (here we may take a real number whose usual partial quotients are bounded), then for the values of the quadratic form $Q\left(z_{1}, z_{2}\right)=\left(z_{1}-\lambda z_{2}\right) z_{2}$, it may be seen that 0 is an isolated point of the set of values of $Q$ over the set $\mathfrak{G}^{2}$ of pairs of Gaussian integers.

In [7] we described a class of real binary quadratic forms for which the values on the set of pairs of integers is dense in $\mathbb{R}$; this is related to a classical result of E. Artin on orbits of the geodesic flow associated to the modular surface. In [7. Artin's result was strengthened, and an application was made to values of the binary quadratic forms over the set of pairs of integers, and also over the set of pairs of positive integers. 
In this section, using complex continued fractions, we describe an analogous class of complex binary quadratic forms for which the set of values on pairs of Gaussian integers is a dense subset of $\mathbb{C}$.

For $u=\left(\begin{array}{l}\alpha_{1} \\ \alpha_{2}\end{array}\right)$ and $v=\left(\begin{array}{l}\beta_{1} \\ \beta_{2}\end{array}\right)$, we denote by $Q_{u, v}$ the quadratic form on $\mathbb{C}^{2}$ defined by

$$
Q_{u, v}\left(z_{1} e_{1}+z_{2} e_{2}\right)=\left(\alpha_{1} z_{1}+\alpha_{2} z_{2}\right)\left(\beta_{1} z_{1}+\beta_{2} z_{2}\right) \text { for all } z_{1}, z_{2} \in \mathbb{C} .
$$

We denote by $\eta: \mathbb{C}^{2} \backslash\{0\} \rightarrow \mathbb{P}$ the canonical quotient map. We note the following fact which can be proved in the same way as Proposition 5.1 of [7; from the statement below we shall mainly be interested in the choice $E=\mathfrak{G}^{2}=\left\{a e_{1}+b e_{2} \mid\right.$ $a, b \in \mathfrak{G}\}$, the subgroup of $\mathbb{C}^{2}$ consisting of pairs of Gaussian integers. We note that $\mathfrak{G}^{2}$ is invariant under the linear action of $S L(2, \mathfrak{G})$ on $\mathbb{C}^{2}$.

Proposition 8.1. Let $u, v, u^{\prime}, v^{\prime} \in \mathbb{C}^{2} \backslash\{0\}$ and suppose that there exists a sequence $\left\{g_{j}\right\}$ in $S L(2, \mathbb{C})$ such that $g_{j}(\eta(u)) \rightarrow \eta\left(u^{\prime}\right)$ and $g_{j}(\eta(v)) \rightarrow \eta\left(v^{\prime}\right)$, as $j \rightarrow \infty$, in $\mathbb{P}$. Let $E$ be a subset of $\mathbb{C}^{2}$ such that ${ }^{t} g_{j}(E) \subseteq E$ for all $j$. Then the closure of the set $Q_{u, v}(E)$ in $\mathbb{C}$ contains $Q_{u^{\prime}, v^{\prime}}(E)$.

Remark 8.2. Consider the quadratic form $Q=Q_{u, v}$ where $u$ and $v$ are linearly independent. We write $u$ and $v$ as $g^{-1}\left(e_{1}\right), g^{-1}\left(e_{2}\right)$ where $g \in G L(2, \mathbb{C})$, so $Q=$ $Q_{g^{-1}\left(e_{1}\right), g^{-1}\left(e_{2}\right)}$. Replacing $Q$ by a scalar multiple we may assume, in studying the question of values over $\mathfrak{G}^{2}$ being dense, $g$ to be in $S L(2, \mathbb{C})$. Let $D$ be the subgroup of $S L(2, \mathbb{C})$ consisting of diagonal matrices. Then we see that for any $d \in D, \eta(u)$ and $\eta(v)$ are fixed under the action of $g^{-1} d g$. If there exist sequences $\left\{\gamma_{j}\right\}$ in $S L(2, \mathfrak{G})$ and $\left\{d_{j}\right\}$ in $D$ such that $\left\{\gamma_{j} g^{-1} d_{j} g\right\}$ is dense in $S L(2, \mathbb{C})$, then applying Proposition 8.1, with $E=\mathfrak{G}^{2}$, we get that the closure of $Q_{u, v}\left(\mathfrak{G}^{2}\right)$ contains $Q_{u^{\prime}, v^{\prime}}\left(\mathfrak{G}^{2}\right)$ for every non-zero pair $\left(u^{\prime}, v^{\prime}\right)$ in $\mathbb{C}^{2}$, which means that $Q_{u, v}\left(\mathfrak{G}^{2}\right)$ is dense in $\mathbb{C}$. We note that in $S L(2, \mathbb{C}),\left\{\gamma_{j} g^{-1} d_{j} g\right\}$ is dense if and only if $\left\{d_{j} g \gamma_{j}\right\}$ is dense. Therefore the condition involved is equivalent to saying that the orbit of the coset of $g$ in $S L(2, \mathbb{C}) / S L(2, \mathfrak{G})$ under the action of $D$ is dense in $S L(2, \mathbb{C}) / S L(2, \mathfrak{G})$. It is well known that $S L(2, \mathfrak{G})$ is a lattice in $S L(2, \mathbb{C})$, viz. $S L(2, \mathbb{C}) / S L(2, \mathfrak{G})$ admits a finite measure invariant under the action of $S L(2, \mathbb{C})$ (see [4, $\S 13$ for a general result in this respect). This implies in particular that the action of $D$ on $S L(2, \mathbb{C}) / S L(2, \mathfrak{G})$ is ergodic (with respect to the $S L(2, \mathbb{C})$-invariant measure); see 3], Chapter III, for instance. Hence for almost all $g$ the above condition holds and consequently the values of $Q=Q_{g^{-1}\left(e_{1}\right), g^{-1}\left(e_{2}\right)}$ over $\mathfrak{G}^{2}$ are dense in $\mathbb{C}$. However, this does not enable one to describe any specific quadratic form for which the conclusion holds. The point about the application here is that we obtain a set of forms which may be explicitly described, in terms of continued fraction expansions, for which this property holds.

We next define the notion of a generic complex number. In this respect we shall restrict ourselves to sequences arising as a continued fraction expansion with respect to the Hurwitz algorithm; the procedure can be extended to other algorithms satisfying suitable conditions, but in the present instance we shall content ourselves considering only the Hurwitz algorithm.

We shall assume that the algorithm is given completely, fixing a convention for tie-breaking when there is more than one Gaussian integer at the minimum distance, so that we can talk unambiguously of the continued fraction expansion of 
any number $z \in C^{\prime}$; for convenience we shall assume the convention to be such that for all $z \in S$, where $S=\left\{z=x+i y|| x\left|\leq \frac{1}{2},\right| y \mid \leq \frac{1}{2}\right\}$ as before, $a_{0}$ is chosen to be 0 . Also, for notational convenience, we shall consider the rational complex numbers $p / q, p, q \in \mathfrak{G}$, to have the expansion $\left\{a_{n}\right\}_{n=0}^{\infty}$ with $\left\{a_{n}\right\}_{n=0}^{m}$ the finite term Hurwitz expansion given by the algorithm, such that $p / q=\omega\left(\left\{a_{n}\right\}_{n=0}^{m}\right)$, and $a_{k}=\infty$ for $k \geq m+1$.

For brevity we shall refer to the corresponding sequence of partial quotients as the Hurwitz expansion of the number. For $z \in S$ and $l \in \mathbb{N}$ we denote by $H_{l}(z)$ the block $\left(a_{1}, \ldots, a_{l}\right)$, where $\left\{a_{n}\right\}$ is the Hurwitz expansion of $z$ (recall that $a_{0}=0$, since $z \in S)$.

For a block $\beta=\left(b_{1}, \ldots, b_{l}\right)$, with $b_{j} \in \mathfrak{G}$ and $\left|b_{j}\right|>1$ for $j=1, \ldots, l$, we denote by $S_{\beta}$ the set of interior points of the set $\left\{z \in S \mid H_{l}(z)=\beta\right\}$. We note that the latter is the intersection of the sets $S, \iota U_{b_{1}}(S), \ldots, \iota U_{b_{1}}(S) \cdots \iota U_{b_{l}}(S)$, where $\iota$ is the map defined by $\iota(z)=z^{-1}$ for all $z \in C^{*}$. Each of these is a subset bounded by four arcs which are segments of a circle or a straight line. We see from this that $S_{\beta}$, if non-empty, is a set whose boundary (not included in $S_{\beta}$ ) consists of finitely many arcs, each a segment of a circle or straight line. Moreover, these segments are images of lines of the form $\operatorname{Re} z=k+\frac{1}{2}$ or $\operatorname{Im} z=k+\frac{1}{2}$, with $k \in \mathbb{Z}$, under fractional transformations of $\mathbb{C}$, of the form $z \mapsto \frac{p z+q}{r z+s}, p, q, r, s \in \mathfrak{G}$ and $p s-q r= \pm 1$.

We say that a block $\beta=\left(b_{1}, \ldots, b_{l}\right)$ is admissible if $S_{\beta}$ is non-empty (it seems plausible that this condition is equivalent to $\left\{z \in S \mid H_{l}(z)=\beta\right\}$ being non-empty; this will however not play a role, and we shall leave it at that).

Remark 8.3. For any block $\beta=\left(b_{1}, \ldots, b_{l}\right)$ let $r_{\beta}=\omega\left(\left\{b_{n}\right\}_{n=0}^{l}\right)$ (notation as introduced $\S 2$ ), with $b_{0}=0$. Then each $r_{\beta}$ is a rational number from $S$; if all $b_{j}, j=1, \ldots, l$, are of absolute value greater than 2 , then $r_{\beta}$ is an interior point of $S_{\beta}$ - otherwise it can be a boundary point of $S_{\beta}$. Let $R$ be the set consisting of all $r_{\beta}$ with $\beta$ any admissible block. Then $R$ is a dense subset of $S$ since for any non-empty open subset $\Omega$ of $S$ we can find a block $\beta$ such that $r_{\beta} \in \Omega$.

We note the following with regard to admissible blocks.

Proposition 8.4. Let $\beta=\left(b_{1}, \ldots, b_{l}\right)$ be an admissible block of Gaussian integers. Then we have the following:

(i) if $\left|b_{l}\right| \geq 2 \sqrt{2}$, then for any admissible block $\beta^{\prime}$ the block $\beta \beta^{\prime}$ is admissible;

(ii) for any $b^{\prime} \in \mathfrak{G}$ with $\left|b^{\prime}\right| \geq 2 \sqrt{2}$ there exists $b \in \mathfrak{G}$ such that $|b|=\left|b^{\prime}\right|$ and $\left(b_{1}, \ldots, b_{l}, b\right)$ is admissible;

(iii) given any admissible block $\beta^{\prime}$ there exists an admissible block $\beta^{\prime \prime}$ such that $\beta$ and $\beta^{\prime}$ are the initial and end blocks of $\beta^{\prime \prime}$; moreover, $l\left(\beta^{\prime \prime}\right)$ can be chosen to be at most $l(\beta)+l\left(\beta^{\prime}\right)+1$.

Proof. (i) When $\left|b_{l}\right| \geq 2 \sqrt{2}$, we have $b_{l}+z \in S^{-1}$ for all $z \in S$, and hence in particular if $\beta$ and $\beta^{\prime}$ are admissible, $S_{\beta \beta^{\prime}}$ has non-empty interior, so $\beta \beta^{\prime}$ is admissible.

(ii) In view of (i) this remains to be proved only when $\left|b_{l}\right| \leq 2$. Suppose $b_{l}=1+i$. Then $b_{l}+z \in S^{-1}$ for $z \in S \backslash(B(-1) \cup B(-i))$, so $\left(b_{1}, \ldots, b_{l}, b\right)$ is admissible for $b \in S^{-1} \backslash\left(B(-1)^{-1} \cup B(-i)^{-1}\right)$, which contains in particular all $b \in \mathfrak{G}$ with $|b| \geq 2 \sqrt{2}$ for which both the real and imaginary coordinates are negative. This shows that (ii) holds for $b_{l}=1+i$. By symmetry the assertion holds whenever $\left|b_{l}\right|=\sqrt{2}$. A similar argument shows that the assertion holds when $\left|b_{l}\right|=2$ as well. 
(iii) If $\left|b_{l}\right| \geq 2 \sqrt{2}$, then by (i) we may take $\beta^{\prime \prime}=\beta \beta^{\prime}$. If $\left|b_{l}\right| \leq 2$, then by (ii) we can find $b$ such that $\beta^{\prime \prime}=\left(b_{1}, \ldots, b_{l}, b\right) \beta^{\prime \prime}$ is admissible, and it has the desired property.

Let $z \in \mathbb{C}^{\prime}$ and $\left\{a_{n}\right\}_{n=0}^{\infty}$ be its Hurwitz expansion; we say that $z$ is generic if every admissible block occurs in $\left\{a_{n}\right\}_{n=0}^{\infty}$. For any admissible block $\beta$ we denote by $E_{\beta}$ the interior of the set of all $z$ in $S$ such that $\beta$ occurs in the Hurwitz expansion $\left\{a_{n}\right\}$ of $z$.

Proposition 8.5. The set of generic complex numbers contains a dense $\mathcal{G}_{\delta}$ subset of $\mathbb{C}$.

Proof. Let $\alpha, \beta$ be any admissible blocks, of lengths $k$ and $l$ respectively. Let $\epsilon>0$ be arbitrary and let $N \in \mathbb{N}$ such that $N>\epsilon^{-1}$. By Proposition 8.4 there exists an admissible block $\gamma=\left(c_{1}, \ldots, c_{m}\right), m>k+l+1$, such that $\alpha$ is an initial block of $\gamma, c_{k+1}=N+2$, and $\beta$ is an end block of $\gamma$. Then $S_{\gamma}$ is contained in $E_{\beta}$. We note that for any $z \in S_{\gamma}$ by Proposition 3.3 we have $\left|z-r_{\alpha}\right| \leq\left(\left|z_{k+1}\right|-1\right)^{-1}$, where $\left\{z_{n}\right\}$ denotes the iteration sequence for $z$ with respect to the Hurwitz algorithm, and we have $\left|z_{k+1}\right| \geq(N+2)-\left|z_{k+2}\right|^{-1}>N+1$. Since $S_{\gamma}$ is contained in $E_{\beta}$, this shows that the distance of $r_{\alpha}$ from $E_{\beta}$ is at most $N^{-1}<\epsilon$. As $\epsilon>0$ is arbitrary this shows that $r_{\alpha}$ is contained in the closure of $E_{\beta}$. Since this holds for every admissible block $\alpha$ it means that the set $R$ as defined in Remark 8.3 is contained in $E_{\beta}$, and since $R$ is dense in $S$ it follows that $E_{\beta}$ is dense in $S$. Thus for every admissible block $\beta$ there exists an open dense subset of $S$ consisting of points such that $\beta$ occurs in their Hurwitz expansion. As there are only countably many blocks we get that there exists a dense $\mathcal{G}_{\delta}$ subset of $S$ consisting of generic complex numbers. Since translates of generic numbers by Gaussian integers are generic, it follows that there exists a dense $\mathcal{G}_{\delta}$ subset of $\mathbb{C}$ all of whose elements are generic complex numbers.

In light of a result of [9] we can also conclude the following.

Proposition 8.6. With respect to the Lebesgue measure on $\mathbb{C}$, almost every $z$ in $\mathbb{C}$ is generic.

Proof. Recall that $S=\left\{z=x+i y|| x\left|\leq \frac{1}{2},\right| y \mid \leq \frac{1}{2}\right\}$. The map $G: S \rightarrow S$ defined by $G(z)=z^{-1}-a_{1} \in S$, where $a_{1}$ is from the Hurwitz expansion $\left\{a_{n}\right\}$ of $z$, is called the Gauss map corresponding to the Hurwitz algorithm (see [9]). We note that $G$ is a non-invertible (many-to-one) measurable map of $S$ into itself. Clearly, if $\left\{a_{n}\right\}_{n=1}^{\infty}$ (with $a_{0}=0$ ) is the Hurwitz expansion of $z$, then the shifted sequence $\left\{a_{n+1}\right\}_{n=1}^{\infty}$ is the Hurwitz expansion of $G(z)$. There exists a probability measure $\mu$ on $S$ equivalent to the Lebesgue measure which is $G$-invariant (namely such that $\mu\left(G^{-1}(E)\right)=\mu(E)$ for all Borel subsets $E$ of $\left.S\right)$ and that the action of $G$ is ergodic with respect to $\mu$; see $[9$, Theorem 5.5 (the action is also mixing, which however will not concern us here). We note that for every admissible block $\beta$ the set $E_{\beta}$ as above is $G$-invariant, in the sense that $G^{-1}\left(E_{\beta}\right) \subseteq E_{\beta}$; since $\mu$ is a finite $G$-invariant measure, this further implies that the symmetric difference $G^{-1}\left(E_{\beta}\right) \Delta E_{\beta}$ is of $\mu$-measure 0 . Since each $E_{\beta}$ is of positive measure, by ergodicity it follows that $\mu\left(E_{\beta}\right)=1$ for all admissible blocks $\beta$. Since $\mu$ is equivalent to the Lebesgue measure, we get that each $E_{\beta}$ is a subset of $S$ with full Lebesgue measure. Hence the set of generic points in $S$, which is the intersection of $E_{\beta}$ over the countable collection of all admissible blocks $\beta$, is of full Lebesgue measure in 
$S$. Since translates of generic points by Gaussian integers are generic, we get that almost every complex number is generic.

We now note the following.

Corollary 8.7. Let $z \in \mathbb{C}$ be a generic complex number with Hurwitz expansion $\left\{a_{n}\right\}$. Let $\alpha, \beta \in \mathbb{C}$ and let $\left\{x_{n}\right\}$ and $\left\{y_{n}\right\}$ be their respective Hurwitz expansions. Suppose that $\left|x_{n}\right|>\sqrt{5}$ for all $n \geq 0,\left|y_{0}\right| \geq \sqrt{2}$, and that all initial blocks of $\left\{y_{n}\right\}$ are admissible. Then there exists a sequence $\left\{g_{j}\right\}$ in $S L(2, \mathfrak{G})$ such that

$$
g_{j}(\pi(\zeta)) \rightarrow \pi(\alpha) \text { for all } \zeta \neq \rho(\pi(z)) \text {, and } g_{j}(\rho(\pi(z))) \rightarrow \rho(\pi(\beta)) \text {. }
$$

Proof. We note that under the conditions assumed in the hypothesis, if $\alpha_{j}$ and $\beta_{j}$ are initial blocks of length $2 j$ in $\left\{x_{n}\right\}$ and $\left\{y_{n}\right\}$ respectively, then $\tilde{\alpha}_{j} \beta_{j}$ are admissible blocks. As $\left\{a_{n}\right\}$ is generic we can find a sequence $\left\{s_{j}\right\}$ in $\mathbb{N}$ such that the conditions in the hypothesis of Proposition [7.5] are satisfied. The assertion of the corollary then follows from Proposition 7.5 .

Remark 8.8. Consider $z \in S$ with Hurwitz expansion $\left\{a_{n}\right\}_{n=0}^{\infty}$, (with $a_{0}=0$ ). If some initial block $\xi=\left(a_{1}, \ldots, a_{l}\right)$ is not admissible, then in particular $z$ is not contained in $S_{\xi}$ and this means that it is contained in one of the boundary segments of $S_{\xi}$ (which as recalled earlier are segments of circles or straight lines). It therefore follows that for almost all $z$ in $S$ all initial blocks of the Hurwitz expansion of $z$ are admissible, and in particular the set is dense in $S$. It therefore follows that the closure of the set of $\beta$ in $\mathbb{C}$ for which the condition in the hypothesis of Corollary 8.7 holds contains $\bigcup_{a \in \mathfrak{G},|a| \geq \sqrt{2}} a+S$; in particular, it contains the annular region consisting of all $z$ in $\mathbb{C}$ with $|z| \geq \sqrt{5 / 2}$.

Corollary 8.9. Let $\zeta_{1}, \zeta_{2} \in \mathbb{C}$ and suppose that $\zeta_{1}$ (or $\left.\zeta_{2}\right)$ is generic. Then the values of the quadratic form $Q(z, w)=\left(z-\zeta_{1} w\right)\left(z-\zeta_{2} w\right)$ over the set of Gaussian integers form a dense subset of $\mathbb{C}$; namely, $\left\{\left(a-\zeta_{1} b\right)\left(a-\zeta_{2} b\right) \mid a, b \in \mathfrak{G}\right\}$ is dense in $\mathbb{C}$.

Proof. Let $v_{r}=\left(\begin{array}{c}\zeta_{r} \\ 1\end{array}\right)$ for $r=1,2$. We shall show that for the quadratic form $Q=Q_{\rho\left(v_{1}\right), \rho\left(v_{2}\right)}$ on $\mathbb{C}^{2}, Q\left(\mathfrak{G}^{2}\right)$ is dense in $\mathbb{C}$. This is readily seen to hold in the case when $\zeta_{1}=\zeta_{2}$; we shall now assume that $\zeta_{1} \neq \zeta_{2}$. We have $\eta\left(v_{1}\right)=\pi\left(\zeta_{1}\right), \eta\left(v_{2}\right)=$ $\pi\left(\zeta_{2}\right)$ and $\eta\left(v_{1}\right) \neq \eta\left(v_{2}\right)$. Let $\alpha, \beta \in \mathbb{C}$ be such that the conditions in the statement of Corollary 8.7 are satisfied. Then by Corollary 8.7 there exists a sequence $\left\{g_{j}\right\}$ in $S L(2, \mathfrak{G})$ such that $g_{j}\left(\rho\left(\eta\left(v_{1}\right)\right)\right) \rightarrow \rho\left(\pi\left(v_{\beta}\right)\right)$ and $g_{j}\left(\eta\left(v_{2}\right)\right) \rightarrow \pi\left(v_{\alpha}\right)$. Then by Proposition 8.6 the closure of $Q\left(\mathfrak{G}^{2}\right)$ in $\mathbb{C}$ contains $Q_{\rho\left(v_{\beta}\right), v_{\alpha}}\left(\mathfrak{G}^{2}\right)$, for all $\alpha, \beta \in \mathbb{C}$ as above. By Remark 8.8 the condition in Corollary 8.7 is satisfied for all $\beta$ in a dense subset of $\{z \in \mathbb{C}|| z \mid \geq \sqrt{5 / 2}\}$. We fix an $\alpha$ for which the condition in Corollary 8.7 is satisfied and consider values $Q_{\rho\left(v_{\beta}\right), v_{\alpha}}\left(\mathfrak{G}^{2}\right)$, for $\beta$ varying in $\{z \in \mathbb{C}|| z \mid \geq \sqrt{5 / 2}\}$. It is straightforward to see that these values contain a dense set of complex numbers. Hence $Q\left(\mathfrak{G}^{2}\right)$ is dense in $\mathbb{C}$.

Remark 8.10. The set of all (complex) binary quadratic forms may be viewed in a natural way as the 3 -dimensional vector space (over $\mathbb{C}$ ) of symmetric $2 \times 2$ matrices, and may be considered equipped with the Lebesgue measure. From the fact that the generic numbers in $\mathbb{C}$ form a set of full Lebesgue measure it may be seen that the quadratic forms as in the statement of Corollary 8.9 and their scalar multiples 
together form a set of full measure in the space of all binary quadratic forms; our conclusion shows that for each of these forms the set of values over the set of pairs $\{(a, b) \mid a, b \in \mathfrak{G}\}$ is a dense subset of $\mathbb{C}$.

\section{ACKNOWLEDGEMENTS}

The first-named author would like to thank the Institut de Mathématiques de Luminy, Marseille, France and Centre National de Recherche Scientifique, France for their hospitality and support while this work was done. The authors would also like to thank the referee for many suggestions leading to improvements in the presentation of the paper.

\section{REFERENCES}

[1] E. Artin, Quadratische Körper im Gebiete der höheren Kongruenzen. I (German), Math. Z. 19 (1924), no. 1, 153-206, DOI 10.1007/BF01181074. MR 1544651

[2] Alan F. Beardon and Ian Short, The Seidel, Stern, Stolz and Van Vleck theorems on continued fractions, Bull. Lond. Math. Soc. 42 (2010), no. 3, 457-466, DOI 10.1112/blms/bdq006. MR2651941 (2011j:40007)

[3] M. Bachir Bekka and Matthias Mayer, Ergodic theory and topological dynamics of group actions on homogeneous spaces, London Mathematical Society Lecture Note Series, vol. 269, Cambridge University Press, Cambridge, 2000. MR:1781937 (2002c:37002)

[4] Armand Borel, Introduction aux groupes arithmétiques (French), Publications de l'Institut de Mathématique de l'Université de Strasbourg, XV. Actualités Scientifiques et Industrielles, No. 1341, Hermann, Paris, 1969. MR0244260 (39 \#5577)

[5] Armand Borel and Gopal Prasad, Values of isotropic quadratic forms at S-integral points, Compositio Math. 83 (1992), no. 3, 347-372. MR1175945 (93j:11022)

[6] Alexander N. Starkov, Dynamical systems on homogeneous spaces, Translations of Mathematical Monographs, vol. 190, American Mathematical Society, Providence, RI, 2000. Translated from the 1999 Russian original by the author. MR.1746847 (2001m:37013b)

[7] S. G. Dani and Arnaldo Nogueira, On orbits of $\mathrm{SL}(2, \mathbb{Z})_{+}$and values of binary quadratic forms on positive integral pairs, J. Number Theory 95 (2002), no. 2, 313-328. MR.1924105 (2003j:11039)

[8] G. H. Hardy and E. M. Wright, An introduction to the theory of numbers, 6th ed., Oxford University Press, Oxford, 2008. Revised by D. R. Heath-Brown and J. H. Silverman; with a foreword by Andrew Wiles. MR 2445243 (2009i:11001)

[9] Doug Hensley, Continued fractions, World Scientific Publishing Co. Pte. Ltd., Hackensack, NJ, 2006. MR2351741 (2009a:11019)

[10] A. Hurwitz, Über die Entwicklung complexer Grössen in Kettenbrüche (German), Acta Math. 11 (1887), no. 1-4, 187-200, DOI 10.1007/BF02418048. MR 1554754

[11] W. J. LeVeque, Continued fractions and approximations in $k(i)$. I, II, Nederl. Akad. Wetensch. Proc. Ser. A. 55 = Indagationes Math. 14 (1952), 526-535, 536-545. MR0053972 $(14,850 \mathrm{~b})$

[12] G. A. Margulis, Oppenheim conjecture, Fields Medallists' lectures, World Sci. Ser. 20th Century Math., vol. 5, World Sci. Publ., River Edge, NJ, 1997, pp. 272-327. MR1622909 (99e:11046)

[13] Asmus L. Schmidt, Diophantine approximation of complex numbers, Acta Math. 134 (1975), 1-85. MR0422168 (54 \#10160)

School of Mathematics, Tata Institute of Fundamental Research, Homi Bhabha Road, Mumbai 400 005, India

E-mail address: dani@math.tifr.res.in

Current address: Department of Mathematics, Indian Institute of Technology Bombay, Powai, Mumbai 400 076, India

AiX-Marseille Université, Institut de Mathématiques de Luminy, 163, Avenue De Luminy, Case 907, 13288 Marseille Cedex 9, France

E-mail address: arnaldo.nogueira@univ-amu.fr 